\title{
Exotic meson-meson molecules and compact four-quark states
}

\author{
J. Vijande, ${ }^{1,2}$ A. Valcarce, ${ }^{2}$ and N. Barnea ${ }^{3,4}$ \\ ${ }^{1}$ Departamento de Fúsica Atómica, molecular y Nuclear, \\ Universidad de Valencia (UV) and IFIC (UV-CSIC), Valencia, Spain. \\ ${ }^{2}$ Departamento de Física Fundamental, \\ Universidad de Salamanca, E-37008 Salamanca, Spain \\ ${ }^{3}$ The Racah Institute of Physics, The Hebrew University, 91904, Jerusalem, Israel \\ ${ }^{4}$ Institute for Nuclear Theory, University of Washington, Seattle, WA 98195, USA
}

(Dated: March 17, 2009)

\begin{abstract}
We present an exact calculation of $S$ and $P$ wave $Q Q \bar{n} \bar{n}$ states using different standard nonrelativistic quark-quark potentials. We explore in detail the charm and bottom sectors looking for bound states that could be measured within existing facilities. Against the proliferation of four-quark states sometimes predicted in the literature, we found a small number of candidates to be stable. We analyze their properties in a trial to distinguish between compact and molecular states. Possible decay modes are discussed.
\end{abstract}

PACS numbers: 12.39.Jh,14.40.Lb,21.45.+v,31.15.Ja 


\section{INTRODUCTION}

The existence of stable $Q Q \bar{n} \bar{n}$ states has been the topic for discussion since the early 80's [1]. These states are of particular interest since they are manifestly exotic, i.e., heavy flavor quantum number \pm 2 with baryon number equal 0 . If they would lie below the threshold for dissociation into two ordinary hadrons they would be narrow and should show up clearly in the experimental spectrum. There are already estimates of the production rates indicating they could be produced and detected at present (and future) experimental facilities [2]. Four-quark states seem to be necessary to tame the bewildering landscape of new meson states. Non-exotic four-quark states embedded in the meson spectra have been proposed as a thoughtful explanation of the proliferation and peculiar properties of light scalar-isoscalar or open-charm mesons [3]. There are recent indications of the possible existence of molecular four-quark states, suggested from the observation of the $Z^{+}(4430)$ [4]. A step forward scrutinizing the structure of low-energy hadrons would be to demonstrate the existence of stable manifestly exotic multiquark states, the $Q Q \bar{n} \bar{n}$ system being an ideal candidate.

To illustrate the theoretical landscape, we present in Table I a summary of different approaches to the manifestly exotic four-quark spectroscopy [5, 6, 7, 8, 19, 10, 11, 12, 13, 14, 15, 16, 17]. Exotic multiquarks were examined in Ref. [5] solving the four-body problem by three different variational methods with a nonrelativistic potential considering explicitly virtual meson-meson components in the wave function. In Ref. [6] four-quark states were studied using a variational approach with trial wave functions whose interaction energies are approximately given by known hadron masses. Ref. 7] used a potential derived from the MIT bag model in the Born-Oppenheimer approximation. The calculations were done by means of the Green's function Monte Carlo method. $b \bar{n}-b \bar{n}$ molecules loosely bound by the one-pion exchange were obtained in Ref. [8] using chiral perturbation theory. Ref. [9] analyzed $L=0$ four-quark systems with the Bhaduri potential and a variational method in a harmonic oscillator basis up to $N=8$ quanta. Ref. [10] discussed the stability of multiquark systems using different parametrizations of a Goldstone boson exchange model and a variational formalism with gaussian trial wave functions. In Ref. [1] the Bhaduri potential was reexamined by means of a variational method that allows nonzero internal orbital angular momentum in the subsystems of quarks and antiquarks. The existence of a shallow tetraquark state $c c \bar{u} \bar{d}$ was discussed in Ref. [12] using semiempirical mass relations. Ref. [13] designed a powerful method, similar to the stochastic variational approach [18], accommodating two free-meson asymptotic states. It was applied to the Bhaduri potential, fixing the results of Refs. [9, 11]. Ref. [14] analyzed multiquark states with a variational formalism using gaussian trial wave functions with only quadratic terms in the Jacobi coordinates. QCD sum rules were used in Ref. [15] to study the possible existence of an axial diquark-antidiquark bound state. In Ref. [16] the ground state of tetraquarks were evaluated assuming a diquark-antidiquark structure, reducing the relativistic four-body problem to the solution of two relativistic two-body problems. Ref. [17] discussed the possible existence of four-quark bound states within the framework of the chiral $S U(3)$ quark model by means of a variational approach using gaussian trial wave functions.

As seen in Table I, there is a remarkable agreement on the existence of a $I=0, J^{P}=1^{+}$ $b b \bar{n} \bar{n}$ bound state and also, although not so neatly, on the existence of a $c c \bar{n} \bar{n}$ one (along this work $n$ stands for a light $u$ or $d$ quark). However, among the different theoretical approaches only a few had paid attention to other quantum numbers, trying to elucidate if a proliferation 
of four-quark states is predicted. Hence, in this work our purpose will be twofold. On the one hand, we shall try to shed some light on this topic by making a detailed analysis of the $Q Q \bar{n} \bar{n}$ spectra discussing each set of quantum numbers. On the other hand, the Achilles' heel of almost all the approaches described above is the lack of an exact numerical method to solve the four-body problem. Most of these works rely on variational calculations with different types of trial wave functions or on semiempirical mass relations. The importance of this problem requires numerical methods able to provide exact solutions with controlled numerical uncertainties.

To this end we shall use a new approach based on the hyperspherical formalism recently developed to solve exactly the four-quark problem [19, 20]. The idea is to perform an expansion of the trial wave function in terms of the hyperspherical harmonics $(\mathrm{HH})$ functions. This allows to generalize the simplicity of the spherical harmonic expansion for the angular functions of a single particle motion to a system of particles by introducing a global length $\rho$, the hyperradius, and a set of angles, $\Omega$. For the $\mathrm{HH}$ expansion to be practical, the evaluation of the potential energy matrix elements must be feasible. The main difficulty of this method is to construct $\mathrm{HH}$ functions with proper permutational symmetry for a system of identical particles. This is a difficult problem that may be overcome by means of the $\mathrm{HH}$ formalism based on the symmetrization of the $N$-body wave function with respect to the symmetric group using the Barnea and Novoselsky algorithm [21]. For systems containing only two pairs of identical particles this problem is greatly simplified making the appropriate choice of Jacobi coordinates. In Ref. [20] we have developed the HH formalism for the $Q \bar{Q} n \bar{n}$ system, and we will use it here with the appropriate modifications to study the exotic $Q Q \bar{n} \bar{n}$ four-quark spectra.

The manuscript is organized as follows. In Sec. II we briefly revise the $\mathrm{HH}$ formalism for the $Q Q \bar{n} \bar{n}$ system and present the quark models used. In Sec. III we introduce observables that may allow to distinguish between unbound and compact or molecular four-quark bound states. In Sec. IV] the results and the analysis of the $c c \bar{n} \bar{n}$ and $b b \bar{n} \bar{n}$ spectroscopy are presented. In Sec. $\mathrm{V}$ the possibility of measuring the predicted bound states within current experimental facilities is discussed. Finally, we summarize in Sec. VI our conclusions.

\section{TECHNICAL DETAILS}

Within the HH expansion, the four-quark wave function can be written as a sum of outer products of color, isospin, spin and configuration terms

$$
\left.\left|\phi_{C I S R}\right\rangle=\mid \text { Color }\right\rangle|\operatorname{Isospin}\rangle[|\operatorname{Spin}\rangle \otimes|R\rangle]^{J M},
$$

such that the four-quark state is a color singlet with well defined parity, isospin and total angular momentum. In the following we shall assume that particles 1 and 2 are the $Q$-quarks and particles 3 and 4 are the $n$-quarks. Thus, particles 1 and 2 are identical, and so are 3 and 4. Consequently, the Pauli principle leads to the following conditions,

$$
\hat{P}_{12}\left|\phi_{C I S R}\right\rangle=\hat{P}_{34}\left|\phi_{C I S R}\right\rangle=-\left|\phi_{C I S R}\right\rangle
$$

$\hat{P}_{i j}$ being the permutation operator of particles $i$ and $j$.

Coupling the color states of two quarks (antiquarks) can yield two possible representations, the symmetric 6-dimensional, $6(\overline{6})$, and the antisymmetric 3 -dimensional, $\overline{3}(3)$. 
Coupling the color states of the quark pair with that of the antiquark pair must yield a color singlet. Thus, there are only two possible color states for a $Q Q \bar{q} \bar{q}$ system [22],

$$
\mid \text { Color }\rangle=\left\{\left|\overline{3}_{12} 3_{34}\right\rangle,\left|6_{12} \overline{6}_{34}\right\rangle\right\} \text {. }
$$

These states have well defined symmetry under permutations, Eq. (2). The spin states with such symmetry can be obtained in the following way,

$$
\mid \text { Spin }\rangle=\left|\left(\left(s_{1}, s_{2}\right) S_{12},\left(s_{3}, s_{4}\right) S_{34}\right) S\right\rangle=\left|\left(S_{12} S_{34}\right) S\right\rangle .
$$

The same holds for the isospin, $\mid$ Isospin $\rangle=\left|\left(i_{3}, i_{4}\right) I_{34}\right\rangle$, which applies only to the $n$-quarks, thus $I=I_{34}$.

We use the $\mathrm{HH}$ expansion to describe the spatial part of the wave function. We choose for convenience the $H$-type Jacobi coordinates,

$$
\begin{aligned}
\boldsymbol{\eta}_{1} & =\mu_{1,2}\left(\boldsymbol{r}_{2}-\boldsymbol{r}_{1}\right) \\
\boldsymbol{\eta}_{2} & =\mu_{12,34}\left(\frac{m_{3} \boldsymbol{r}_{3}+m_{4} \boldsymbol{r}_{4}}{m_{34}}-\frac{m_{1} \boldsymbol{r}_{1}+m_{2} \boldsymbol{r}_{2}}{m_{12}}\right), \\
\boldsymbol{\eta}_{3} & =\mu_{3,4}\left(\boldsymbol{r}_{4}-\boldsymbol{r}_{3}\right)
\end{aligned}
$$

were $m_{i j}=m_{i}+m_{j}, \mu_{i, j}=\sqrt{m_{i} m_{j} / m_{i j}}$, and $m_{1234}=m_{1}+m_{2}+m_{3}+m_{4}$. Using these vectors, it is easy to obtain basis functions that have well defined symmetry under permutations of the pairs (12) and (34). In the $\mathrm{HH}$ formalism the three Jacobi vectors are transformed into a single length variable, $\rho=\sqrt{\eta_{1}^{2}+\eta_{2}^{2}+\eta_{3}^{2}}$, and 8-angular variables, $\Omega$, that represent the location on the 8-dimensional sphere. The spatial basis states are given by

$$
\langle\rho \Omega \mid R\rangle=U_{n}(\rho) \mathcal{Y}_{[K]}(\Omega),
$$

were $\mathcal{Y}_{[K]}$ are the $\mathrm{HH}$ functions, and $[K] \equiv\left\{K K_{12} L M_{L} L_{12} \ell_{3} \ell_{2} \ell_{1}\right\}$. The quantum number $K$ is the grand angular momentum, $L M_{L}$ are the usual orbital angular momentum quantum numbers, and $\ell_{i}$ is the angular momentum associated with the Jacobi vector $\boldsymbol{\eta}_{i}$. The quantum numbers $K_{12}, L_{12}$ correspond to the intermediate coupling of $\boldsymbol{\eta}_{1}$ and $\boldsymbol{\eta}_{2}$. The Laguerre functions are used as the hyper-radial basis functions $U_{n}(\rho)$.

The Pauli principle, Eq. (2), leads to the following restrictions on the allowed combinations of basis states:

(i) $(-1)^{S_{12}+\ell_{1}}=+1,(-1)^{S_{34}+I+\ell_{3}}=-1$ for the $\left|6_{12} \overline{6}_{34}\right\rangle$ color state,

(ii) $(-1)^{S_{12}+\ell_{1}}=-1,(-1)^{S_{34}+I+\ell_{3}}=+1$ for the $\left|\overline{3}_{12} 3_{34}\right\rangle$ state.

Assuming non-relativistic quantum mechanics we solve the four-body Schrödinger equation using the basis states described above. The grand angular momentum $K$ is the main quantum number in our expansion and the calculation is truncated at some $K$ value.

For our study we will use two standard quark potential models. The constituent quark cluster (CQC) model was proposed in the early 90's in an attempt to obtain a simultaneous description of the nucleon-nucleon interaction and the baryon spectra [23]. Later on it was generalized to all flavor sectors giving a reasonable description of the meson [24] and baryon spectra [25]. The model contains Goldstone boson exchange between quarks, a one-gluonexchange (OGE) potential, and a screened confined interaction as dictated by unquenched lattice calculations [26]. The model parameters have been taken from Ref. [24] with the 
FIG. 1: $H$-type Jacobi vectors.

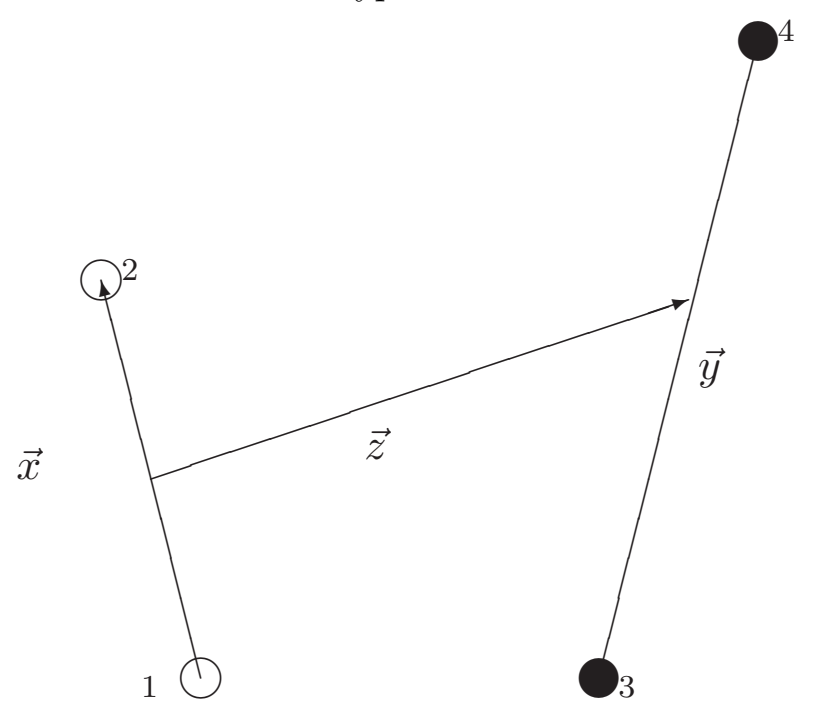

exception of the OGE regularization parameter, see Ref. [20] for details. In the following we shall denote this parametrization as CQC and the standard parametrization of Ref. [24] will be referred to as $\mathrm{CQC}_{18}$. Explicit expressions of the interacting potentials and a detailed discussion of the model can be found in Ref. [24]. The Bhaduri-Cohler-Nogami (BCN) model was proposed in the early 80's in an attempt to obtain a unified description of meson and baryon spectroscopy [27]. It was later on applied to study the baryon spectra [28] and fourquark systems [9]. The model retains the most important terms of the one-gluon exchange (OGE) interaction, namely coulomb and spin-spin, and a linear confining potential. The parameters are taken from Ref. [9].

A summary of the energies obtained with both models, CQC and BCN, for selected meson states that may appear in the thresholds of the studied four-quark systems are given in Table II, together with the corresponding experimental energies.

\section{BOUND STATES, COMPACT AND MOLECULAR}

\section{A. Threshold determination}

As thoroughly discussed in Ref. [20], in order to discriminate between four-quark bound states and simple pieces of the meson-meson continuum, one has to carefully determine the two-meson states that constitute the thresholds for each set of quantum numbers. Dealing with strongly interacting particles, the two-meson states should have well defined total angular momentum $(J)$, parity $(P)$, and a properly symmetrized wave function if two identical mesons are considered (spin-statistics theorem). When noncentral forces are not taken into account, orbital angular momentum $(L)$ and total spin $(S)$ are also good quantum numbers. We give in Tables III, IV], V] VI, and VII the lowest threshold in both cases, that we will refer to as coupled (CO) and uncoupled (UN) schemes respectively, together with the final state relative orbital angular momentum of the decay products. We would like to emphasize that although we use central forces in our calculation the $\mathrm{CO}$ scheme is the relevant one for observations, since a small non-central component in the potential is enough to produce a 
sizeable effect on the width of a state. In Table III we summarize the thresholds obtained using the experimental energies given in Ref. [29]. In Tables [V] and $\mathrm{V}$ we quote the thresholds obtained with the CQC model for the charm and bottom sectors respectively, and in Tables VI and VII those for the BCN model.

An important property of the $Q Q \bar{n} \bar{n}$ system, that is crucial for the possible existence of bound states, is the fact that only one physical threshold $(Q \bar{n})(Q \bar{n})$ is allowed. Consequently, particular modifications of the four-quark interaction, for instance a strong color-dependent attraction in the $Q Q$ pair, would not be translated into any asymptotically free two-meson state. As discussed in Ref. [31], this is not a general property in the four-quark spectroscopy, as the $Q \bar{Q} n \bar{n}$ four-quark state has two allowed physical thresholds: $(Q \bar{Q})(n \bar{n})$ and $(Q \bar{n})(n \bar{Q})$.

\section{B. Figures of merit}

The relevant quantity for analyzing the stability of any four-quark state is $\Delta_{E}$, the energy difference between the mass of the four-quark system and that of the lowest two-meson threshold,

$$
\Delta_{E}=E_{4 q}-E\left(M_{1}, M_{2}\right),
$$

where $E_{4 q}$ stands for the four-quark energy and $E\left(M_{1}, M_{2}\right)$ for the energy of the two-meson threshold. Thus, $\Delta_{E}<0$ indicates that all fall-apart decays are forbidden, and therefore one has a proper bound state. $\Delta_{E} \geq 0$ will indicate that the four-quark solution corresponds to an unbound threshold (two free mesons).

One of the main difficulties in studying four-quark states, already discussed in Refs. [19, 20], is the slow convergence of unbound solutions. We show in Fig. 2 the evolution of $E_{4 q} / E\left(M_{1}, M_{2}\right)$ as a function of $K$ for three different states, two of them bound (solid and dash-dotted lines) and one unbound (dashed line). Although all of them converge for large enough values of $K$, the correct description of the two-meson threshold for unbound states is slow and time consuming. A helpful tool to minimize this problem was proposed in Ref. [19] through an extrapolation of the four-quark energy using the expression

$$
E_{4 q}(K)=E_{4 q}(K=\infty)+\frac{a}{K^{b}},
$$

where $E_{4 q}(K=\infty), a$ and $b$ are fitted parameters. When this extrapolation is used for unbound states, one can observe how the four-quark energies reproduce the thresholds to within a few $\mathrm{MeV}$.

A second important quantity to characterize the possible existence of a bound state is the evolution of the root mean square radius as a function of $K$. While for unbound states, the components of the four-quark state will tend to be far away when increasing $K$, for a bound state the radius should stabilize when increasing $K$. In order to compare four-quark against two free-meson states we define the root mean square radius for four (two) quark systems

$$
\operatorname{RMS}_{4 q(2 q)}=\left(\frac{\sum_{i=1}^{4(2)} m_{i}\left\langle\left(r_{i}-R\right)^{2}\right\rangle}{\sum_{i=1}^{4(2)} m_{i}}\right)^{1 / 2}
$$

and its corresponding ratio

$$
\Delta_{R}=\frac{\mathrm{RMS}_{4 q}}{\mathrm{RMS}_{M_{1}}+\mathrm{RMS}_{M_{2}}}
$$


FIG. 2: $E_{4 q} / E\left(M_{1}, M_{2}\right)$ as a function of $K$ for the $J^{P}(L, S, I)=1^{+}(0,1,0)$ (solid line), $0^{+}(0,0,0)$ (dashed line), and $1^{-}(1,0,1)$ (dash-dotted line) with the CQC model.

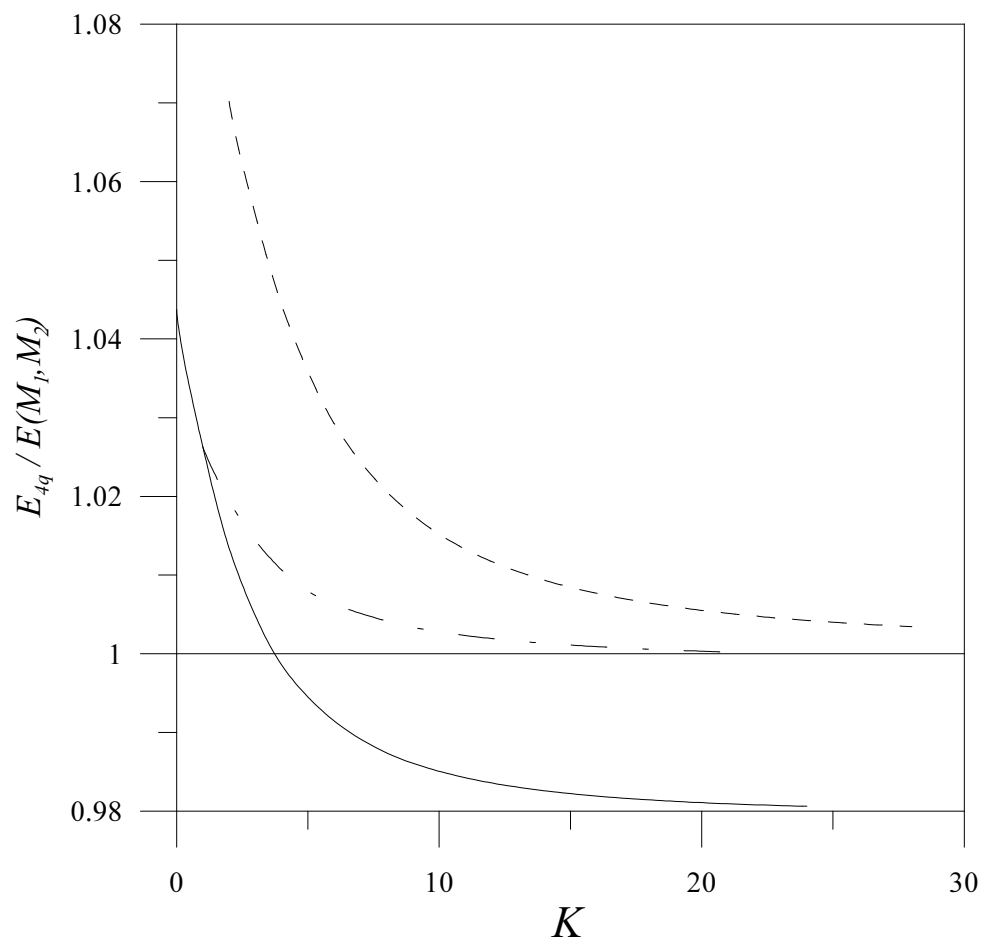

where $\mathrm{RMS}_{M_{1}}+\mathrm{RMS}_{M_{2}}$ stands for the sum of the radii of the mesons corresponding to the lowest threshold.

\section{Compact vs molecular states}

Besides trying to unravel the possible existence of bound $Q Q \bar{n} \bar{n}$ states one should try to understand whether it is possible to differentiate between compact and molecular states. A molecular state may be understood as a four-quark state containing a single physical two-meson component, i.e., a unique singlet-singlet component in the color wave function with well-defined spin and isospin quantum numbers. One could expect these states not being deeply bound and therefore having a size of the order of the two-meson system, i.e., $\Delta_{R} \sim 1$. Opposite to that, a compact state may be characterized by its involved structure on the color space, its wave function containing different singlet-singlet components with non negligible probabilities. One would expect such states would be smaller than typical two- 
meson systems, i.e., $\Delta_{R}<1$. Let us notice that while $\Delta_{R}>1$ but finite would correspond to a meson-meson molecule $\Delta_{R} \stackrel{K \rightarrow \infty}{\longrightarrow} \infty$ would represent an unbound threshold.

One may illustrate the situation described above by the deuteron and the $H$-dibaryon examples. Let us try to draw the analogy between these states and the $Q Q \bar{n} \bar{n}$ system. The deuteron has a small binding energy of $-2.225 \mathrm{MeV}$ and a ratio between its root mean square charge radius $(2.139 \mathrm{fm})$ and the one of two protons $(1.75 \mathrm{fm})$ of $1.222[32], \Delta_{R}=1.22$ and $\Delta_{E}=-2.225 \mathrm{MeV}$ in our notation. Should the deuteron be considered as a pure baryonbaryon molecule? Although Ref. 33] emphasized the difficulties to identify pure hadronhadron molecules close to thresholds, in the deuteron case it was long-ago justified [34]. The probability of physical two-baryon states other than nucleon-nucleon is meaningless [23]. Therefore it constitutes a clear example of a molecular state. The postulated $H$-dibaryon would however fit into the picture of compact states, its wave function presenting relevant components of different singlet-singlet physical channels: $\Lambda \Lambda, N \Xi$, and $\Sigma \Sigma$ at least [35].

This last aspect makes contact with the role played by hidden-color configurations, color singlets built by nonsinglet constituents. There are three different ways for coupling two quarks and two antiquarks in a colorless state,

$$
\begin{aligned}
& {\left[\left(q_{1} q_{2}\right)\left(\bar{q}_{3} \bar{q}_{4}\right)\right]=\left\{\left|\overline{3}_{12} 3_{34}\right\rangle,\left|6_{12} \overline{6}_{34}\right\rangle\right\}} \\
& {\left[\left(q_{1} \bar{q}_{3}\right)\left(q_{2} \bar{q}_{4}\right)\right]=\left\{\left|1_{13} 1_{24}\right\rangle,\left|8_{13} 8_{24}\right\rangle\right\}} \\
& {\left[\left(q_{1} \bar{q}_{4}\right)\left(q_{2} \bar{q}_{3}\right)\right]=\left\{\left|1_{14} 1_{23}\right\rangle,\left|8_{14} 8_{23}\right\rangle\right\} .}
\end{aligned}
$$

Each coupling scheme allows to define a color basis where the four-body problem can be solved. The first basis, $\left[\left(q_{1} q_{2}\right)\left(\bar{q}_{3} \bar{q}_{4}\right)\right]$, being the most suitable one to deal with the Pauli principle, is made entirely by hidden-color vectors. The other two are hybrid basis that contain both singlet-singlet (physical) and octect-octect (hidden-color) components. It is possible to prove from simple group theory arguments that once we have solved the four-body problem for a system composed of two identical quarks $(Q Q)$ and two identical antiquarks $(\bar{n} \bar{n})$, there is a minimum value for the octect-octect component probability of the wave function either in the $\left[\left(Q_{1} \bar{n}_{3}\right)\left(Q_{2} \bar{n}_{4}\right)\right]$ or the $\left[\left(Q_{1} \bar{n}_{4}\right)\left(Q_{2} \bar{n}_{3}\right)\right]$ couplings: $P_{88}^{13,24}, P_{88}^{14,23} \in$ $[1 / 3,2 / 3]$. It can also be proved that for a four-quark threshold state $P_{88}^{13,24}=P_{88}^{14,23}=4 / 9$. Does this imply an important hidden-color component in all $Q Q \bar{n} \bar{n}$ states? The answer is no. In Ref. [36] it was proved than any physical state can be expanded in terms of a basis constructed by direct product of mesonic and/or baryonic states not necessarily linearly independent. Therefore one can express any $Q Q \bar{n} \bar{n}$ state in terms of the singlet-singlet component of the $\left[\left(Q_{1} \bar{n}_{3}\right)\left(Q_{2} \bar{n}_{4}\right)\right]$ and $\left[\left(Q_{1} \bar{n}_{4}\right)\left(Q_{2} \bar{n}_{3}\right)\right]$ basis.

This discussion can be made more quantitative. Let us assume that $\{P, Q\}$ and $\{\hat{P}, \hat{Q}\}$ are the projectors associated to two orthonormal basis that are not orthogonal to each other, i.e., $P \hat{P}|\phi\rangle \neq 0$ and $P \hat{Q}|\phi\rangle \neq 0$ for an arbitrary state $|\phi\rangle$. This would be the case of the two orthonormal basis: $\left\{\left|1_{13} 1_{24}\right\rangle,\left|8_{13} 8_{24}\right\rangle\right\}$ and $\left\{\left|1_{14} 1_{23}\right\rangle,\left|8_{14} 8_{23}\right\rangle\right\}$. Any arbitrary state can be written as

$$
|\Psi\rangle=P|\Psi\rangle+Q|\Psi\rangle
$$

and the probability of the state associated to $P$ or $\hat{P}$ will be given by [38],

$$
\begin{aligned}
\mathcal{P}^{|\Psi\rangle}([u]) & =\frac{1}{2\left(1-\cos ^{2} \alpha\right)}[\langle\Psi|P \hat{Q}| \Psi\rangle+\langle\Psi|\hat{Q} P| \Psi\rangle] \\
\mathcal{P}^{|\Psi\rangle}\left(\left[u^{\prime}\right]\right) & =\frac{1}{2\left(1-\cos ^{2} \alpha\right)}[\langle\Psi|\hat{P} Q| \Psi\rangle+\langle\Psi|Q \hat{P}| \Psi\rangle]
\end{aligned}
$$


where $P=|u\rangle\langle u|$ and $\hat{P}=\left|u^{\prime}\right\rangle\left\langle u^{\prime}\right|$ and $\cos \alpha=\left\langle u^{\prime} \mid u\right\rangle$. For a molecular state either $\mathcal{P}^{|\Psi\rangle}([u])$ or $\mathcal{P}^{|\Psi\rangle}\left(\left[u^{\prime}\right]\right)$ would be equal to zero while for a compact state both will be different from zero.

\section{RESULTS}

\section{A. Comparison to other numerical methods}

To illustrate the performance of the numerical procedure described in Sec. II it is convenient to compare with other numerical methods to understand its capability and advantages, if any. As outlined in Sec. I, in the past decades there have been several attempts to study multiquarks containing explicit charm or bottom flavors. Among them we shall analyze the calculation of the $(L, S, I)=(0,1,0) c c \bar{n} \bar{n}$ state of Refs. [9, 13] using the BCN potential and the results obtained in Ref. [14] with the CQC model.

We present in Table VIII results for different $L=0$ spin-isospin $c c \bar{n} \bar{n}$ states within the CQC model. We quote in the first column the results obtained with a variational calculation using gaussian trial wave functions with only quadratic terms in the Jacobi coordinates [14] ( $S$ wave approximation). Such approximation is recovered in our formalism requiring $\ell_{i}=0$ for all pairs. These results are quoted in the second column up to $K=10$, being fully converged, and reproducing exactly the variational results ${ }^{1}$. The relevance of large relative orbital angular momenta can be judged by looking at the last column, where almost exact $\mathrm{HH}$ results up to $K=24$ are given, the difference in some cases being larger than $200 \mathrm{MeV}$. This effect was not appreciated in Ref. [14] since their importance was estimated using only one gaussian for the radial wave function.

Using the variational method of Ref. [37], where nonzero relative orbital angular momenta were considered, we have obtained $3861.38 \mathrm{MeV}$ for the energy and $0.363 \mathrm{fm}$ for the root mean square radius, respectively, of the $(L, S, I)=(0,1,0) \operatorname{cc} \bar{n} \bar{n}$ four-quark state. This result is in perfect agreement with that obtained using the $\mathrm{HH}$ formalism: $3860.65 \mathrm{MeV}$ and $0.367 \mathrm{fm}$.

We have also reproduced the calculation of the $(L, S, I)=(0,1,0) \operatorname{cc} \bar{n} \bar{n}$ state of Refs. [9, 13] using the BCN model. In Table IX (second and third columns) we present the energies and RMS obtained for this state for all values of $K$. For $K=26$ we have obtained an energy of 3899.2 MeV as compared to 3904.7 MeV of Ref. [13] and 3931.0 MeV of Ref. [9]. Ref. [13] designed a powerful method similar to the stochastic variational approach to study this particular system. Although they are not fully converged, the agreement gives confidence on both results. Ref. [9] uses a diagonalization in a restricted Hilbert space, obtaining a larger value.

\section{B. The $Q Q \bar{n} \bar{n}$ system}

In Ref. [20] the question: Does the quark model naturally predict the existence of $Q \bar{Q} n \bar{n}$ bound states? was posed. The answer was clear, no compact bound four-quark state were found for any set of quantum numbers if only two-body potentials in a complete basis

\footnotetext{
${ }^{1}$ We have redone the calculation of Ref. [14] with the parameters used in this work for a proper comparison.
} 
were used. One cannot discard that a modification of the Hilbert space, like for example considering only diquark configurations, or of the interacting hamiltonian, like many-body contributions, could give rise to bound states. Is the same conclusion still valid in the $Q Q \bar{n} \bar{n}$ sector? To answer this question we have performed an exhaustive analysis of the $Q Q \bar{n} \bar{n}$ spectra by means of the quark models described above. Some particular results were already presented in Ref. [31], where we studied the possibility of the $X(3872)$ being a $c \bar{c} n \bar{n}$ tetraquark. We gave arguments that favored the existence of $Q Q \bar{n} \bar{n}$ stable states in nature, while making harder the existence of $Q \bar{Q} n \bar{n}$ stable states. To make the physics clear we compared two particular sets of quantum numbers: $J^{P C}(I)=1^{++}(0)$ for $c \bar{c} n \bar{n}$ and $J^{P}(I)=1^{+}(0)$ for $c c \bar{n} \bar{n}$. Based on a variational study with a confining mass independent many body potential we argued in Refs. [31, 40] that the binding would increase when increasing the mass ratio of flavor exotic four-quark systems. Definitive conclusions can only be obtained based on realistic calculations. For these purposes, in the present work we have considered all isoscalar and isovector states with total orbital angular momentum $L \leq 1$. For positive (negative) parity four-quark systems the ground state corresponds to $L=0(L=1)$, since parity can be expressed in terms of the relative orbital angular momenta associated to the Jacobi coordinates as $P=(-)^{\ell_{1}+\ell_{2}+\ell_{3}}$. This means that $P=-1$ needs three units of relative orbital angular momentum to obtain $L=0\left(\ell_{1}=\ell_{2}=\ell_{3}=1\right)$ while only one is needed for $L=1$. The same reasoning applies for $P=+1$ states. Therefore, since the complexity of the calculation and the computing time increase with $L$, we have not considered $L=1$ positive parity states that should be higher in energy. The calculation has been done up to the maximum value of $K$ within our computational capabilities, $K_{\max }$.

In Table $\mathrm{X}$ we present the $c c \bar{n} \bar{n}$ CQC final results obtained for all possible $L=0$ isoscalar and isovector states and for the negative parity $L=1$ states. We indicate for each state the maximum value of $K$ used, $K_{\max }$. A first glance at this table sheds two general conclusions. Firstly, opposite to the $Q \bar{Q} n \bar{n}$ case there exist bound states in the $Q Q \bar{n} \bar{n}$ spectra when only two-body potentials are used. Second, once the four-body problem is properly solved the number of bound states is small. Curiously, there are two cases that do not converge to the lowest possible two-meson threshold but to a higher one, the $(L=0, I=0) J^{P}=0^{-}$and $2^{-}$. These states get stuck in the lowest $D$ wave threshold, either the $\left.D_{1} D_{2}\right|_{D}(5316 \mathrm{MeV})$ or the $\left.D_{J}^{*} D_{J^{\prime}}^{*}\right|_{D}(5325 \mathrm{MeV})$, satisfying $\Delta_{E} \geq 0$.

As discussed in Sec. III the convergence of unbound or molecular states close to the two meson threshold is a difficult numerical problem. In order to minimize this problem we have introduced the extrapolation formula (8). In Table XI, we compare the energies obtained for $K_{\max }$ and for $K \rightarrow \infty$, using the extrapolation formula. For unbound or loosely bound states we observe how the extrapolation leads to four-quark energies within only a few $\mathrm{MeV}$ of the corresponding threshold. Throughout this manuscript we shall always refer to $E_{4 q}\left(K_{\max }\right)$ as the four-quark state energy, $E_{4 q}$. In particular cases we will use the extrapolation to study the characteristics of some specific states, mainly those that are close below the threshold.

There are two ingredients that may alter the stability of the $Q Q \bar{n} \bar{n}$ system: either the mass of the heavy quark or the interacting potential. One should wonder if the characteristic spectrum obtained in Table $\mathrm{X}$ would be greatly influenced by them. It was pointed out in the early 80 's that a $Q Q \bar{Q}^{\prime} \bar{Q}^{\prime}$ four-quark state should be stable against dissociation into $Q \bar{Q}^{\prime}+Q \bar{Q}^{\prime}$ if the ratio $m_{Q} / m_{Q^{\prime}}$ is large enough [1]. This was corroborated by chiral perturbation theory and lattice QCD studies of the $b b \bar{n} \bar{n}$ system [8, 39]. Trying to disentangle if a proliferation of states is predicted when the mass of the heavy quark augments, we studied all ground states of the bottom sector using the same interacting potential as above. The 
results are presented in Table XII. We observe that all bound states become deeper than in the charm sector and a few new states appear. Our results strengthen the conclusion that the larger the ratio of the quark masses the larger the binding energies.

The second ingredient to be tested is the dependence of the results on the particular choice of the quark-quark interaction. To answer this question we have reanalyzed all the predicted bound states using the BCN model. The results are summarized in Tables XIII and XIV] The existence of bound states is also evident when the BCN model is considered, although the characteristics of each state depend on the model considered. Of particular interest is the observation that the bottom sector presents, independently of the quark model, bound states with binding energies of the order of $100 \mathrm{MeV}$ that should be possible to observe.

In connection with the interacting potential used, it has been recently analyzed in Ref. [40] the stability of $Q Q \bar{n} \bar{n}$ and $Q \bar{Q} n \bar{n}$ systems in a simple string model considering only a multiquark confining interaction given by the minimum of a flip-flop or a butterfly potential in an attempt to discern whether confining interactions not factorizable as two-body potentials would influence the stability of four-quark states. The ground state of systems made of two quarks and two antiquarks of equal masses was found to be below the dissociation threshold. While for the cryptoexotic $Q \bar{Q} n \bar{n}$ the binding decreases when increasing the mass ratio $m_{Q} / m_{n}$, for the flavor exotic $Q Q \bar{n} \bar{n}$ the effect of mass symmetry breaking is opposite. Although more realistic calculations are needed before establishing a definitive conclusion, the findings of Ref. [40] corroborate our results.

\section{Charm sector}

In the following we will study more closely those $c c \bar{n} \bar{n}$ quantum numbers, $J^{P}(L, S, I)$, that may host a bound state $\left(\Delta_{E}<0\right.$ in Tables $\mathrm{X}$ and XIII) trying to unveil states that might be a consequence of model and computational approximations. Expected bound states are summarized in Table XV.

$$
\text { 1. } 1^{+}(0,1,0)
$$

The possible existence of a four-quark state with these quantum numbers was predicted more than twenty years ago by Zouzou and collaborators [5]. Since then, several works have been devoted to study these particular quantum numbers by means of different methods and interactions, either in the charm or in the bottom sector [6, 7, 10, 11, 12, 13, 15]. In Table IX we show the results obtained in this work with the BCN and CQC models. In both cases the state converges below threshold. For the CQC model the predicted binding energy is large, $-76 \mathrm{MeV}$, and $\Delta_{R}<1$, what would fit into the defined compact states. Opposite to that, the BCN model predicts a rather small binding, $-7 \mathrm{MeV}$, and the RMS is larger than one but still not converged, although not increasing linearly. This state would naturally correspond to a molecule.

Although this state would be stable against dissociation into two mesons, it may decay electromagnetically or weakly. The electromagnetic transition $(Q Q \bar{n} \bar{n}) \rightarrow(Q \bar{n})(Q \bar{n}) \gamma$ would be allowed if $E_{4 q}$ is larger than the mass of two $D$ mesons. The process is illustrated in the upper part of Figure 3. For the CQC model the four-quark energy is below the $D D$ threshold and therefore the predicted state could only decay via a second order weak process into either two kaons (Cabibbo allowed) or two light mesons (Cabibbo suppressed). 
The process is illustrated in the bottom part of Figure 3. Opposite to that, the results of the BCN model allow for an electromagnetic decay with the emission of a photon with an energy lower than $127 \mathrm{MeV}$ in the $c c \bar{n} \bar{n}$ rest frame.

2. $(1,2,3)^{-}(1,2,1)$ and $1^{-}(1,0,1)$

Bound states have been obtained either with the CQC or the BCN models or with both. In these cases a detailed analysis of the possible thresholds is required. As previously noticed, the interacting models only consider central terms, $L$ and $S$ being proper quantum numbers. However, as illustrated is Sec. III A the thresholds may be different in the coupled or uncoupled schemes, the former one being the relevant when trying to compare with experiment. We show in the upper part of Table XVI the results obtained compared to the threshold in the uncoupled and coupled schemes. The differences are noticeable, when the coupled scheme is used both interacting models give results above the corresponding lowest threshold, what discard these quantum numbers as promising candidates for being observed experimentally.

As already discussed in Sec. IVB, increasing the mass of the heavy quark will favor the binding. This is illustrated in Fig. 4 comparing the four-quark energy of the $1^{-}(1,0,1)$ state to its uncoupled threshold in the CQC model. As can be seen the CQC model predicts a tiny binding energy, but the extrapolation gives a very stable value around $\Delta_{E} \approx-6 \mathrm{MeV}$. Opposite to the case of unbound states, the RMS does not grow linearly with $K$. We observe the changes in $\Delta_{E}$ when increasing $m_{Q}$, noting how $\Delta_{E}$ crosses zero for masses slightly below the charm quark mass. As a consequence, all the states discussed in this section might be candidates to be stable in the bottom sector due to the binding gained with the heavy quark mass.

\section{Bottom sector}

We repeat the same analysis as before for the bottom sector. Expected bound states are summarized in Table XV.

\section{1. $1^{+}(0,1,0)$}

Opposite to the charm sector, in the bottom sector both quark models offer the same prediction, a compact deeply bound four-quark state. Within the CQC model the binding energy gets a value of $-214 \mathrm{MeV}$ while BCN predicts a somewhat smaller value of $-144 \mathrm{MeV}$. Concerning the possible decays, both of them are also below the threshold for electromagnetic decays into $B B$ (10588 MeV for CQC and $10602 \mathrm{MeV}$ for $\mathrm{BCN}$ ) and therefore the only allowed decay mode for this state will involve a second order weak process into two opencharm (Cabibbo allowed) or two light mesons (Cabibbo suppressed).

2. $0^{+}(0,0,0)$ and $3^{-}(1,2,1)$

The existence of a positive parity $Q Q \bar{n} \bar{n}$ bound state with quantum numbers $S=0$ and $I=0$ was proposed in Ref. [5] using the BCN model if the ratio between the masses of the 
heavy and light quarks was larger than 5-10, i.e., $b c \bar{n} \bar{n}$ and heavier. We have obtained a similar limit for the CQC model, $m_{b} / m_{n} \approx 15$.

For the $J^{P}=3^{-}$state a bound state has also been found using both models, being $\Delta_{E}=-140 \mathrm{MeV}$ and $\Delta_{E}=-119 \mathrm{MeV}$, respectively. As can be seen in Table XVI it is the only member of a multiplet below all possible thresholds and therefore all strong decays are forbidden.

No bound states were observed in the charm sector with these quantum numbers, being therefore consequence of the binding gained due to the larger heavy quark mass. Both states would present electromagnetic decays, the former one $(b b \bar{n} \bar{n}) \rightarrow\left(\left.B B\right|_{P}\right) \gamma$, with a photon energy in the range of $\lesssim 400 \mathrm{MeV}$, and the latter through $(b b \bar{n} \bar{n}) \rightarrow\left(\left.B B\right|_{D}\right) \gamma$ or $(b b \bar{n} \bar{n}) \rightarrow\left(\left.B^{*} B^{*}\right|_{S}\right) \gamma$ emitting a photon of the order of $\lesssim 400-450 \mathrm{MeV}$.

3. $1^{-}(1,0,0)$

Although no bound state is observed in the $c c \bar{n} \bar{n}$ spectra, a bound state, $\Delta_{E}=-11 \mathrm{MeV}$, appears in the bottom sector with the CQC model. The structure of the wave function, $\Delta_{R} \approx 1.182$, clearly points to an extended meson-meson molecule instead of a compact four-quark state. Concerning its possible decay channels, being this state below all possible thresholds it could only undergo a weak decay.

$$
\text { 4. } 1^{-}(1,0,1), 2^{+}(0,2,1) \text { and }(1,2)^{-}(1,2,1)
$$

All these states are predicted to be bound in the uncoupled scheme but none of them survive the coupled thresholds. Although interesting from the theoretical point of view to carefully test our calculating framework, they are not expected to be observed in nature.

\section{E. Beyond $Q Q \bar{n} \bar{n}$}

Once the $Q Q \bar{n} \bar{n}(Q=c, b)$ states have been discussed, few avenues remain to be explored. Among them, the consideration of $Q Q \bar{s} \bar{s}$ and $Q Q^{\prime} \bar{n} \bar{n}$ states would be of interest. Concerning the strangeness \pm 2 states only those containing $b$ quarks seem likely candidates to accommodate bound states. We have redone the calculation for the most promising states, those with quantum numbers $I\left(J^{P}\right)=0\left(1^{+}\right)$and $Q=c$ or $b$. In both cases the four-quark system is above the corresponding threshold. This can be easily understood because the mass ratio $M_{Q} / m_{q}$ has diminished due to the large mass of the strange quark, thus increasing the contribution of the kinetic energy. Besides, the attractive one-pion exchange does not contribute. Our conclusions coincide with Ref. [16], the binding energy decreases when decreasing the mass ratio $M_{Q} / m_{q}$. The second system, $Q Q^{\prime} \bar{n} \bar{n}$ is made of distinguishable heavy quarks, i.e., $b c \bar{n} \bar{n}$ among others, and therefore demands a modification of the current formalism, namely consider all possible combinations of $\ell_{1}, S_{12}$. This would lead to an increase in the basis size and consequently in computing time. However, one can draw a simple conclusion in light of Ref. [16], or the first six lines of Table III. The binding energy increases with the reduced mass of the heavy quark pair. Thus, one would expect a bound state with quantum numbers $1^{+}(0,1,0)$ for the $b c \bar{n} \bar{n}$ system, while the others appearing in the doubly-bottom sector would not be clear candidates for bound states with a $b c$ heavy diquark. 


\section{F. Meson-meson probabilities}

We present in Table XVII the meson-meson probabilities for some selected four-quark states according to Eqs. (13). These calculations where done by means of the variational method, Ref. [37]. As can be seen there is a perfect agreement with the HH results but the variational formalism allows to evaluate the probabilities of the different physical components in a simpler manner. Unbound states converge to two isolated mesons, the lowest threshold of the system, its RMS and $\Delta_{R}$ being very large. In contrast, bound states have a radius smaller than the threshold and they present probabilities different from zero for several physical states, the lowest two-meson threshold being contained in the physical four-quark system. Such states would be called compact in our notation. When the binding energy approaches the threshold, the probability of a single physical channel converges to one, what we defined as a molecular state.

\section{G. BCN vs. CQC}

The delicate interplay between the OGE and the chiral interactions in the description of the hadron spectra and baryon-baryon interaction has been widely discussed in the literature [42]. In the $Q Q \bar{n} \bar{n}$ spectra the relative strength of these interactions is even more important since $\Delta_{E}$ is very sensitive to any modification in the chiral/OGE rate. The reason is that the threshold is not affected by the chiral part of the interaction since it is made of two $Q \bar{n}$ mesons, and there are no boson exchanges between heavy and light quarks. This is not the case for the $Q Q \bar{n} \bar{n}$ systems, where bosons may be exchanged between the two light antiquarks. Therefore, an arbitrary modification in the strength of the chiral part of the interaction in any four-quark state where it is attractive, could bind the system.

We therefore emphasize the importance of testing any model against as many observables as possible in order to constraint its parameters and ingredients. In this respect the heavylight four-quark states are ideally suited for this task. Since only the total energy, and not the threshold, depends on the boson exchanges, the comparison between the predicted and measured $\Delta_{E}$ would provide us with precise information regarding the role played by these interactions in the meson spectra.

Amazingly, as observed in Table XV almost all bound states are predicted independently of the dynamics. In other words, whenever a bound state is found with the CQC model, the BCN potential predicts a similar state. In general predicted binding energies are smaller for gluon-based interactions. Only the $1^{-}(1,0,0) b b \bar{n} \bar{n}$ state is predicted by one of the models, the CQC. To illustrate the larger binding predicted by models considering boson exchanges we have selected one of the states whose binding energy in the uncoupled scheme has been found to be smaller than $10 \mathrm{MeV}$, the $b b \bar{n} \bar{n} 2^{+}(0,2,1)$. Once boson exchanges are switched off the state lacks of enough attraction to be bound, behaving like two isolated mesons. These results are illustrated in Table XVIII. To illustrate the difference in the structure induced by the boson exchanges we have plotted in Fig. 5 the evolution with $K$ of $\Delta_{R}$ in both cases. In the unbound case the system is separating very rapidly while the bound one starts to converge to a large, but finite, value. 


\section{EXPERIMENTAL OBSERVATION}

The most promising mechanism for the production of a four-quark state is the independent formation of two $c c$ and $\bar{q} \bar{q}$ pairs to later on merge into a $c c \bar{q} \bar{q}$ state. Following this idea the rate of $c c \bar{q} \bar{q}$ production was estimated in Ref. [12] as $R_{c c \bar{q} \bar{q}} / R_{c c q} \approx 1 / 10$. Therefore, any facility able to produce double charm baryons in sizable quantities should be able, in principle, to observe four-quark states.

So far, double charmed baryons have only been observed by SELEX Collaboration [43], although its limited statistics, $\approx 50$ events, makes doubtful that four-quark states can be produced in quantities large enough to be statistically significant. In Ref. [44] the production rate of double charmed baryons by the COMPASS experiment was estimated to be of the order of $10^{4}$ to $1.7 \cdot 10^{4}$ events, this will indicate that up to 1500 four-quark events may be produced by COMPASS $200 \mathrm{GeV}$ proton beam [45]. The number of $c c q$ and $b b q$ events at Tevatron was estimated in Ref. [46], obtaining values of $10^{5}$ and $10^{4}$ events respectively. This would yield values of the order of $10^{4}$ four-quark events to be produced in the second run of Tevatron. Although so far Belle Collaboration has not reported the observation of double charmed baryons, their production cross section was estimated in Ref. [47], where values of the order of $10^{4}$ events/year were obtained. This will translate into 1000 fourquark events/year. Theoretical predictions about the production cross-section of double charmed baryons at BaBar has been estimated, giving diverse values from hundreds to tens of thousands of events [48]. This will indicate that BaBar could produce up to 5000 four-quark/events. On this respect it is worth to mention that theoretical cross-section predictions for double $c \bar{c}$ production have been found to be one order of magnitude too low as compared with experimental data [49]. This will imply that the predicted rate for double charmed baryon production may have been underestimated and therefore the expected number of four-quark events could be larger. In Ref. [2], the $c c \bar{q} \bar{q}$ production rate at different facilities was estimated assuming that the dominant mechanism for double charm production at high energy colliders would be a disconnected double gluon-gluon fusion, $(g+g)+(g+g) \rightarrow(c+\bar{c})+(c+\bar{c})$. The predicted number of four-quark events produced at LHC by either LHCb or ALICE and at Tevatron is found to be very large, 9700, 20900, and 600 events/hour respectively, while for RHIC they expect a smaller value of 12 events/hour.

The picture that seems to emerge from these estimations is that nowadays we are on the verge where actual experimental facilities may be capable of start disentangling the properties of $c c \bar{q} \bar{q}$ bound states. If this is not so, the future facilities that will be in operation in the next decade, if not sooner, at CERN and Fermilab will be able to provide a definitive answer to the existence of four-quark flavor-exotic states in nature.

\section{SUMMARY}

In this work we have performed a systematic analysis of all $c c \bar{n} \bar{n}$ and $b b \bar{n} \bar{n}$ ground states within the framework of the hyperspherical harmonic method. In order to distinguish between unbound, compact, and molecular four-quark states we have considered two different quark models widely used in the literature. We have analyzed both isoscalar and isovector systems with $S=0,1$, and 2 . We have considered $L=0$ states with positive and negative parity and $L=1$ negative parity ones. The relevance of a careful analysis of the numerical thresholds together with the numerical approximations involved has been emphasized in order to avoid the misidentification of bound states. Estimations about the possibility to 
detect these states on the next generation of experimental facilities have been performed.

Our results are summarized in Table XV. We have found five four-quark states that should be narrow and therefore possible to detect. Four of them are predicted independently of the interacting potential used, either CQC or BCN. The $1^{-}(1,0,0)$ state is found only with the CQC model. The $c c \bar{n} \bar{n}$ system would only have one bound state while up to four could be stable in the $b b \bar{n} \bar{n}$ system. All predicted states are compact, only the $1^{-}(1,0,0)$ being molecular. Unfortunately, only one of them, the $c c \bar{n} \bar{n}$ state with quantum numbers $1^{+}(0,1,0)$, is within the scope of the experimental facilities that will be available in the near future.

Theoretical models point out to the existence of a double charmed isoscalar four-quark bound state with quantum numbers $J^{P}=1^{+}$, its properties depending on the quark model considered. The experimental detection and analysis of four-quark double charmed states will undoubtedly prove to be an invaluable testing ground to severely constraint the different theoretical models and therefore, it will allow to refine the theoretical predictions all over the hadronic spectra.

\section{ACKNOWLEDGMENTS}

JV thanks M. Moinester for stimulating discussions regarding double charm production. This work has been partially funded by the Spanish Ministerio de Educación y Ciencia and EU FEDER under Contract No. FPA2007-65748, by Junta de Castilla y León under Contracts No. SA016A17 and Grupos de Excelencia GR12, and by the Spanish ConsoliderIngenio 2010 Program CPAN (CSD2007-00042).

[1] J. P. Ader, J. M. Richard, and P. Taxil, Phys. Rev. D 25, 2370 (1982); J. L. Ballot and J. M. Richard, Phys. Lett. B 123, 449 (1983).

[2] A. del Fabbro, D. Janc, M. Rosina, and D. Treleani, Phys. Rev. D 71, 014008 (2005).

[3] C. Amsler and N. A. Tornqvist, Phys. Rep. 389, 61 (2004).

[4] Belle Collaboration, K. Abe et al., Phys. Rev. Lett. 100, 142001 (2008).

[5] S. Zouzou, B. Silvestre-Brac, C. Gignoux, and J. M. Richard, Z. Phys. C 30, 457 (1986).

[6] H. J. Lipkin, Phys. Lett. B 172, 242 (1986).

[7] L. Heller and J. A. Tjon, Phys. Rev. D 35, 969 (1987); J. Carlson, L. Heller, and J. A. Tjon, Phys. Rev. D 37, 744 (1988).

[8] A. V. Manohar and M. B. Wise, Nucl. Phys. B399, 17 (1993).

[9] B. Silvestre-Brac and C. Semay, Z. Phys. C 57, 273 (1993); ibid 61, 271 (1994).

[10] S. Pepin, Fl. Stancu, M. Genovese, and J. M. Richard, Phys. Lett. B 393, 119 (1997).

[11] D. M. Brink and Fl. Stancu, Phys. Rev. D 57, 6778 (1998).

[12] B. A. Gelman and S. Nussinov, Phys. Lett. B 551, 296 (2003).

[13] D. Janc and M. Rosina, Few-Body Systems 35, 175 (2004).

[14] J. Vijande, A. Valcarce, F. Fernández, and B. Silvestre-Brac, Eur. Phys. J. A 19, 383 (2004).

[15] F. S. Navarra, M. Nielsen, and S. -H. Lee, Phys. Lett. B 649, 166 (2007).

[16] D. Ebert, R. N. Faustov, V. O. Galkin, and W. Lucha, Phys. Rev. D 76, 114015 (2007).

[17] M. Zhang, H. -X. Zhang, and Z. -Y. Zhang, arXiv:0711.1029.

[18] K. Varga and Y. Suzuki, Phys. Rev. C 52, 2885 (1995). 
[19] N. Barnea, J. Vijande, and A. Valcarce, Phys. Rev. D 73, 054004 (2006).

[20] J. Vijande, E. Weissman, N. Barnea, and A. Valcarce, Phys. Rev. D 76, 094022 (2007).

[21] N. Barnea and A. Novoselsky, Ann. Phys. (N. Y.) 256, 192 (1997); Phys. Rev. A 57, 48 (1998).

[22] R. L. Jaffe, Phys. Rev. D 15, 267 (1977); ibid 281 (1977).

[23] A. Valcarce, H. Garcilazo, F. Fernández, and P. González, Rep. Prog. Phys. 68, 965 (2005), and references therein.

[24] J. Vijande, F. Fernández, and A. Valcarce, J. Phys. G 31, 481 (2005).

[25] A. Valcarce, H. Garcilazo, and J. Vijande, Phys. Rev. C 72, 025206 (2005); H. Garcilazo, J. Vijande, and A. Valcarce, J. Phys. G 34, 961 (2007), Eur. Phys. J. A 37, 217 (2008).

[26] G. S. Bali, Phys. Rep. 343, 1 (2001).

[27] R. K. Bhaduri, L. E. Cohler, and Y. Nogami, Nuovo Cimento A65, 376 (1981).

[28] B. Silvestre-Brac and C. Gignoux, Phys. Rev. D 32, 743 (1985).

[29] W. -M. Yao et al., J. Phys. G 33, 1 (2006).

[30] Belle Collaboration, K. Abe et al., Phys. Rev. D 69, 112002 (2004).

[31] J. Vijande, E. Weissman, A. Valcarce, and N. Barnea, Phys. Rev. D 76, 094027 (2007).

[32] P. J. Mohr and B. N. Taylor, Rev. Mod. Phys. 77, 1 (2005).

[33] R. L. Jaffe, Prog. Theor. Phys. Suppl. 168, 127 (2007).

[34] S. Weinberg, Phys. Rev. 65, 137 (1965).

[35] T. Sakai, K. Shimizu, and K. Yazaki, Prog. Theor. Phys. Supp. 137, 121 (2000).

[36] M. Harvey, Nucl. Phys. A 352, 301 (1981); P. González and V. Vento, Few-Body Systems 2, 145 (1987).

[37] J. Vijande, A. Valcarce, F. Fernández, and B. Silvestre-Brac, Phys. Rev. D 72, 034025 (2005).

[38] R. S. Manning, and N. De Leon, J. Math. Chem. 5, 323 (1990).

[39] C. Michael and P. Pennanen, Phys. Rev. D 60, 054012 (1999).

[40] J. Vijande, A. Valcarce, and J. M. Richard, Phys. Rev. D 76, 114013 (2007).

[41] S. Fleck and J. M. Richard, Prog. Theor. Phys. 82, 760 (1989).

[42] C. Nakamoto and H. Toki, Prog. Theor. Phys. 99, 1001 (1998); M. Furuichi and K. Shimizu, Phys. Rev. C65, 025201 (2002); L. A. Blanco, F. Fernández, and A. Valcarce, Phys. Rev. C59, 428 (1999).

[43] SELEX Collaboration, M. Mattson et al., Phys. Rev. Lett. 89, 112001 (2002).

[44] L. Schmitt, S. Paul, R. Kuhn, and M. A. Moinester, hep-ex/0310049.

[45] M. A. Moinester, private comunication.

[46] K. Anikeev et al., hep-ph/0201071.

[47] A. V. Berezhnoy and A. K. Likhoded, Phys. Atom. Nucl. 67, 757 (2004).

[48] A. J. Edwards, Ph.D. dissertation, http://pages.pomona.edu aje04747/thesis.pdf; V. V. Kiselev, A. K. Likhoded, and M. V. Shevlyagin, Phys. Lett. B 332, 411 (1994); V. V. Braguta, V. V. Kiselev, and A. E. Chalov, Phys. Atom. Nucl. 65, 1537 (2002); J. -P. Ma and Z. -G. Si, Phys. Lett. B 568, 135 (2003).

[49] B. Aubert et al., Phys. Rev. D 72, 031101 (2005). 
TABLE I: Energy difference, in $\mathrm{MeV}$, between four-quark states $Q Q \bar{n} \bar{n}(Q=c$ or $b)$ and the corresponding two-meson threshold for different theoretical approaches.

\begin{tabular}{|c|c|c|c|c|c|c|c|c|c|c|c|c|}
\hline & \multicolumn{6}{|c|}{$c c \bar{n} \bar{n}$} & \multicolumn{6}{|c|}{$b b \bar{n} \bar{n}$} \\
\hline & \multicolumn{3}{|c|}{$I=0$} & \multicolumn{3}{|c|}{$I=1$} & \multicolumn{3}{|c|}{$I=0$} & \multicolumn{3}{|c|}{$I=1$} \\
\hline$J^{P}$ & $0^{+}$ & $1^{+}$ & $2^{+}$ & $0^{+}$ & $1^{+}$ & $2^{+}$ & $0^{+}$ & $1^{+}$ & $2^{+}$ & $0^{+}$ & $1^{+}$ & $2^{+}$ \\
\hline$[5]$ & & $>0$ & & & & & & -106 & & & & \\
\hline$[6]$ & & $\lesssim 0$ & & & & & & $<0$ & & & & \\
\hline$[7]$ & & $>0$ & & & & & & $\approx-70$ & & & & \\
\hline$[8]$ & & & & & & & & -8.3 & & & & \\
\hline$[9]$ & $>+60$ & +19 & $>+60$ & $>+60$ & $>+60$ & $>+60$ & $\|>+60$ & -131 & $>+60$ & $>+60$ & +56 & +30 \\
\hline$[10]^{a}$ & & $-185(-332)$ & & & & & & $-226(-497)$ & & & & \\
\hline [11] & & & & & & & & -99 & & +156 & +117 & +86 \\
\hline$[12]$ & & $-(30-60)$ & & & & & & & & & & \\
\hline$[13]^{b}$ & & $-0.6(-2.7)$ & & & & & & $-132(-140)$ & & & & \\
\hline [14] & +585 & -129 & +830 & +384 & +293 & +192 & +258 & -341 & +708 & +128 & +96 & +65 \\
\hline$[15]$ & & $+125 \pm 200$ & & & & & & $-400 \pm 300$ & & & & \\
\hline [16] & & +64 & & +327 & +208 & +104 & & -102 & & +90 & +53 & +23 \\
\hline$[17]^{c}$ & & $+50(+60)$ & & +143 & +299 & +213 & & $-32(-18)$ & & +119 & +93 & +72 \\
\hline
\end{tabular}

${ }^{a}$ Results for two different set of parameters $C_{1}\left(C_{2}\right)$.

${ }^{b}$ Results for two different interacting potentials.

${ }^{c}$ Results for two different interacting potentials. 
TABLE II: $D$ and $B$ meson masses (in MeV) and root mean square radius, RMS, (in fm) obtained with the quark models described in Sec. II] Experimental masses (Exp.) are taken from Ref. [29], except for the state denoted by a dagger that has been taken from Ref. [30].

\begin{tabular}{|cc|c|cc|cc|cc|}
\hline & \multirow{2}{*}{ Exp. } & \multicolumn{2}{c|}{$\mathrm{CQC}_{18}$} & \multicolumn{2}{c|}{ CQC } & \multicolumn{2}{c|}{ BCN } \\
$(L, S, J)$ & State & & $\mathrm{E}$ & $\mathrm{RMS}$ & $\mathrm{E}$ & $\mathrm{RMS}$ & $\mathrm{E}$ & $\mathrm{RMS}$ \\
\hline$(0,0,0)$ & $D$ & $1864.5 \pm 0.4$ & 1883 & 0.207 & 1936 & 0.220 & 1886 & 0.212 \\
$(1,1,1)$ & $D^{*}$ & $2006.7 \pm 0.4$ & 2010 & 0.237 & 2001 & 0.234 & 2020 & 0.235 \\
$(1,0,1)$ & $D_{0}^{*}$ & $2308.0 \pm 17 \pm 12^{\dagger}$ & 2465 & 0.344 & 2498 & 0.373 & 2491 & 0.342 \\
$(1,1,1)$ & $D_{1}^{*}$ & $2422.3 \pm 1.3$ & 2492 & 0.370 & 2490 & 0.369 & 2455 & 0.332 \\
$(1,1,2)$ & $D_{2}^{*}$ & $2461.1 \pm 1.6$ & 2496 & 0.380 & 2498 & 0.373 & 2491 & 0.342 \\
\hline$(0,0,0)$ & $B$ & $5279.0 \pm 0.5$ & 5281 & 0.139 & 5294 & 0.142 & 5301 & 0.141 \\
$(0,1,1)$ & $B^{*}$ & $5325.0 \pm 0.6$ & 5321 & 0.146 & 5318 & 0.145 & 5350 & 0.147 \\
$(1,1,0)$ & $B_{0}^{*}$ & $5698 \pm 8$ & 5848 & 0.230 & 5810 & 0.232 & 5825 & 0.217 \\
$(1,0,1)$ & $B_{1}$ & $5698 \pm 8$ & 5768 & 0.239 & 5807 & 0.231 & 5811 & 0.214 \\
$(1,1,1)$ & $B_{1}^{*}$ & $5698 \pm 8$ & 5876 & 0.232 & 5810 & 0.232 & 5825 & 0.217 \\
$(1,1,2)$ & $B_{2}^{*}$ & $5698 \pm 8$ & 5786 & 0.231 & 5810 & 0.232 & 5825 & 0.217 \\
\hline
\end{tabular}

${ }^{a}$ According to the Particle Data book [29] this signal can be interpreted as stemming from several narrow and broad resonances in the range $5650-5750 \mathrm{MeV}$. No quantum numbers are given. 
TABLE III: Experimental lowest two-meson thresholds for charmed and bottom four-quark states in the uncoupled (UN) and coupled (CO) schemes as defined in the text. $\left.M_{1} M_{2}\right|_{L}$ indicates the lowest threshold and $E\left(M_{1}, M_{2}\right)$ its energy. Energies are in $\mathrm{MeV}$.

\begin{tabular}{|c|c|c|c|c|c|c|c|c|}
\hline & \multicolumn{4}{|c|}{$c c \bar{n} \bar{n}$} & \multicolumn{4}{|c|}{$b b \bar{n} \bar{n}$} \\
\hline & \multicolumn{2}{|c|}{$\overline{\mathrm{UN}}$} & \multicolumn{2}{|c|}{$\mathrm{CO}$} & \multicolumn{2}{|c|}{$\overline{\mathrm{UN}}$} & \multicolumn{2}{|c|}{$\mathrm{CO}$} \\
\hline$J^{P}(I)$ & \multicolumn{2}{|c|}{$\left.M_{1} M_{2}\right|_{L} \quad E\left(M_{1}, M_{2}\right)$} & \multicolumn{2}{|c|}{$\left.M_{1} M_{2}\right|_{L} E\left(M_{1}, M_{2}\right)$} & \multicolumn{2}{|c|}{$\left.M_{1} M_{2}\right|_{L} E\left(M_{1}, M_{2}\right)$} & \multicolumn{2}{|c|}{$\left.M_{1} M_{2}\right|_{L} E\left(M_{1}, M_{2}\right)$} \\
\hline \multicolumn{9}{|c|}{$L=0$} \\
\hline $0^{+}(0)$ & $\overline{\left.D_{1} D\right|_{P}}$ & 4290 & $\left.D_{1} D\right|_{P}$ & 4290 & $\left.B_{1} B\right|_{P}$ & 10977 & $\overline{\left.B_{1} B\right|_{P}}$ & 10977 \\
\hline $0^{+}(1)$ & $\left.D D\right|_{S}$ & 3735 & $\left.D D\right|_{S}$ & 3735 & $\left.B B\right|_{S}$ & 10558 & $\left.B B\right|_{S}$ & 10588 \\
\hline $1^{+}(0,1)$ & $\left.D D^{*}\right|_{S}$ & 3877 & $\left|D D^{*}\right|_{S, D}$ & 3877 & $\left.B B^{*}\right|_{S}$ & 10604 & $\left.B B^{*}\right|_{S, D}$ & 10604 \\
\hline $2^{+}(0)$ & $\left.D^{*} D_{0}^{*}\right|_{P}$ & 4317 & $\left.D D^{*}\right|_{D}$ & 3877 & $\left.B^{*} B_{0}^{*}\right|_{P}$ & 11023 & $\left.B B^{*}\right|_{D}$ & 10604 \\
\hline $2^{+}(1)$ & $\left.D^{*} D^{*}\right|_{S}$ & 4018 & $\left.D D\right|_{D}$ & 3735 & $\left.B^{*} B^{*}\right|_{S}$ & 10650 & $\left.B B\right|_{D}$ & 10558 \\
\hline $0^{-}(0,1)$ & $\left.D_{0}^{*} D_{1}^{*}\right|_{P}$ & 4735 & $\left.D D^{*}\right|_{P}$ & 3877 & $\left.B_{0}^{*} B_{1}^{*}\right|_{P}$ & 11396 & $\left.B B^{*}\right|_{P}$ & 10604 \\
\hline $1^{-}(0)$ & $\left.D_{1} D_{0}^{*}\right|_{P}$ & 4730 & $\left.D D\right|_{P}$ & 3735 & $\left.B_{1} B_{0}^{*}\right|_{P}$ & 11396 & $\left.B B\right|_{P}$ & 10558 \\
\hline $1^{-}(1)$ & $\left.D_{0}^{*} D_{0}^{*}\right|_{P}$ & 4616 & $\left.D D^{*}\right|_{P}$ & 3877 & $\left.B_{0}^{*} B_{0}^{*}\right|_{P}$ & 11396 & $\left.B B^{*}\right|_{P}$ & 10604 \\
\hline $2^{-}(0,1)$ & $\left.D_{0}^{*} D_{1}^{*}\right|_{P}$ & 4735 & $\left.D D^{*}\right|_{P}$ & 3877 & $\left.B_{0}^{*} B_{1}^{*}\right|_{P}$ & 11396 & $\left.B B^{*}\right|_{P}$ & 10604 \\
\hline \multicolumn{9}{|c|}{$L=1 S=0$} \\
\hline $1^{-}(0)$ & $\left.D D\right|_{P}$ & 3735 & $\left.D D\right|_{P}$ & 3735 & $\left.B B\right|_{P}$ & 10558 & $\left.B B\right|_{P}$ & 10558 \\
\hline $1^{-}(1)$ & $\left.D D_{1}\right|_{S, D}$ & 4290 & $\left.D D^{*}\right|_{P}$ & 3877 & $\left.B B_{1}\right|_{S, D}$ & 10977 & $\left.B B^{*}\right|_{P}$ & 10604 \\
\hline \multicolumn{9}{|c|}{$L=1 S=1$} \\
\hline $0^{-}(0,1)$ & $\left.D D^{*}\right|_{P}$ & 3877 & $\left.D D^{*}\right|_{P}$ & 3877 & $\left.B B^{*}\right|_{P}$ & 10604 & $\left.B B^{*}\right|_{P}$ & 10604 \\
\hline $1^{-}(0)$ & $\left.D D^{*}\right|_{P}$ & 3877 & $\left.D D\right|_{P}$ & 3735 & $\left.B B^{*}\right|_{P}$ & 10604 & $\left.B B\right|_{P}$ & 10558 \\
\hline $1^{-}(1)$ & $\left.D D^{*}\right|_{P}$ & 3877 & $\left.D D^{*}\right|_{P}$ & 3877 & $\left.B B^{*}\right|_{P}$ & 10604 & $\left.B B^{*}\right|_{P}$ & 10604 \\
\hline $2^{-}(0,1)$ & $\left.D D^{*}\right|_{P}$ & 3877 & $\left.D D^{*}\right|_{P}$ & 3877 & $\left.B B^{*}\right|_{P}$ & 10604 & $\left.B B^{*}\right|_{P}$ & 10604 \\
\hline \multicolumn{9}{|c|}{$L=1 S=2$} \\
\hline $1^{-}(0)$ & $\left.D^{*} D^{*}\right|_{P}$ & 4018 & $\overline{\left.D D\right|_{P}}$ & 3735 & $\left.B^{*} B^{*}\right|_{P}$ & 10650 & $\overline{\left.B B\right|_{P}}$ & 10558 \\
\hline $1^{-}(1)$ & $\left.D^{*} D_{0}^{*}\right|_{S, D}$ & 4317 & $\left.D D^{*}\right|_{P}$ & 3877 & $\left.B^{*} B_{0}^{*}\right|_{S, D}$ & 11023 & $\left.B B^{*}\right|_{P}$ & 10604 \\
\hline $2^{-}(0)$ & $\left.D^{*} D^{*}\right|_{P}$ & 4018 & $\left.D D^{*}\right|_{P}$ & 3877 & $\left.B^{*} B^{*}\right|_{P}$ & 10650 & $\left.B B^{*}\right|_{P}$ & 10604 \\
\hline $2^{-}(1)$ & $\left.D^{*} D_{0}^{*}\right|_{D}$ & 4317 & $\left.D D^{*}\right|_{P}$ & 3877 & $\left.B^{*} B_{0}^{*}\right|_{D}$ & 11023 & $\left.B B^{*}\right|_{P}$ & 10604 \\
\hline $3^{-}(0)$ & $\left.D^{*} D^{*}\right|_{P}$ & 4018 & $\left.D^{*} D^{*}\right|_{P}$ & 4018 & $\left.B^{*} B^{*}\right|_{P}$ & 10650 & $\left|B^{*} B^{*}\right|_{P}$ & 10650 \\
\hline $3^{-}(1)$ & $\left.D^{*} D_{0}^{*}\right|_{D}$ & 4317 & $\left.D D_{1}\right|_{D}$ & 4290 & $\left.B^{*} B_{0}^{*}\right|_{D}$ & 11023 & $\left.B B_{1}\right|_{D}$ & 10977 \\
\hline
\end{tabular}


TABLE IV: Same as Table III for $c c \bar{n} \bar{n}$ states with the CQC model. We have evaluated the RMS, in $\mathrm{fm}$.

\begin{tabular}{|c|c|c|c|c|c|c|}
\hline & \multicolumn{3}{|c|}{ UN } & \multicolumn{3}{|c|}{$\mathrm{CO}$} \\
\hline$J^{P}(I)$ & $\left.M_{1} M_{2}\right|_{L}$ & $\left(M_{1}, M_{2}\right.$ & RMS & $\left.M_{1} M_{2}\right|_{L}$ & $\left(M_{1}\right.$, & - \\
\hline \multicolumn{7}{|c|}{$L=0$} \\
\hline $0^{+}(0)$ & $\left.D_{1} D\right|_{P}$ & 4426 & 0.589 & $\left.D_{1} D\right|_{P}$ & 4426 & 0.58 \\
\hline $0^{+}(1)$ & $\left.D D\right|_{S}$ & 3872 & 0.440 & $\left.D D\right|_{S}$ & 3872 & 0.44 \\
\hline $1^{+}(0,1)$ & $\left.D D^{*}\right|_{S}$ & 3937 & 0.454 & $\left|D D^{*}\right|_{S, D}$ & 3937 & 0.45 \\
\hline $2^{+}(0)$ & $\left.D^{*} D_{J}^{*}\right|_{P}$ & 4499 & 0.607 & $\left.D D^{*}\right|_{D}$ & 3937 & 0.45 \\
\hline $2^{+}(1)$ & $\left.D^{*} D^{*}\right|_{S}$ & 4002 & 0.468 & $\left.D D\right|_{D}$ & 3872 & 0.44 \\
\hline $0^{-}(0)$ & $\left.D_{1} D_{1}\right|_{P}$ & 4980 & 0.738 & $\left.D D^{*}\right|_{P}$ & 3937 & 0.45 \\
\hline $0^{-}(1)$ & $\left.D_{J}^{*} D_{J}^{*}\right|_{P}$ & 4996 & 0.746 & $\left.D D^{*}\right|_{P}$ & 3937 & 0.45 \\
\hline $1^{-}(0)$ & $\left.D_{1} D_{J}^{*}\right|_{P}$ & 4988 & 0.742 & $\left.D D\right|_{P}$ & 38 & 0.44 \\
\hline $1^{-}(1)$ & $\left.D_{1} D_{J}^{*}\right|_{P}$ & 4988 & 742 & $\left.D D^{*}\right|_{P}$ & $39:$ & 45 \\
\hline $2^{-}(0,1)$ & $\left.D_{J}^{*} D_{J}^{*}\right|_{P}$ & 4996 & 0.746 & $\left.D D^{*}\right|_{P}$ & 3937 & 0.45 \\
\hline \multicolumn{7}{|c|}{$L=1 S=0$} \\
\hline (0) & $\left.D D\right|_{P}$ & 3872 & 440 & $\left.D D\right|_{P}$ & 38 & 0.44 \\
\hline $1^{-}(1)$ & $\left.D D_{1}\right|_{S, D}$ & 4426 & 0.589 & $\left.D D^{*}\right|_{P}$ & 3937 & 0.45 \\
\hline \multicolumn{7}{|c|}{$L=1 S=1$} \\
\hline $0^{-}(0,1)$ & $\left.D D^{*}\right|_{P}$ & 3937 & 0.454 & $\left.D D^{*}\right|_{P}$ & 39 & 0.45 \\
\hline $1^{-}(0)$ & $\left.D D^{*}\right|_{P}$ & 3937 & 0.454 & $\left.D D\right|_{P}$ & 3872 & 0.44 \\
\hline $1^{-}(1)$ & $\left.D D^{*}\right|_{P}$ & 3937 & 0.454 & $\left.D D^{*}\right|_{P}$ & 3937 & 0.45 \\
\hline $2^{-}(0,1)$ & $\left.D D^{*}\right|_{P}$ & 3937 & 0.454 & $\left.D D^{*}\right|_{P}$ & 3937 & 0.454 \\
\hline \multicolumn{7}{|c|}{$L=1 S=2$} \\
\hline $1^{-}(0)$ & $\left.D^{*} D^{*}\right|_{P}$ & 4002 & 0.468 & $\overline{\left.D D\right|_{P}}$ & 3872 & 0.440 \\
\hline $1^{-}(1)$ & $\left.D^{*} D_{J}^{*}\right|_{S, D}$ & 4499 & 0.607 & $\left.D D^{*}\right|_{P}$ & 3937 & 0.45 \\
\hline $2^{-}(0)$ & $\left.D^{*} D^{*}\right|_{P}$ & 4002 & 0.468 & $\left.D D^{*}\right|_{P}$ & 3937 & 0.45 \\
\hline $2^{-}(1)$ & $\left.D^{*} D_{J}^{*}\right|_{S, D}$ & 4499 & 0.607 & $\left.D D^{*}\right|_{P}$ & 3937 & 0.45 \\
\hline $3^{-}(0)$ & $\left.D^{*} D^{*}\right|_{P}$ & 4002 & 0.468 & $\left.D^{*} D^{*}\right|_{P}$ & 4002 & 0.46 \\
\hline $3^{-}(1)$ & $\left.D^{*} D_{J}^{*}\right|_{S, D}$ & 4499 & 0.607 & $\left.D_{1} D\right|_{D}$ & 4426 & 0.58 \\
\hline
\end{tabular}


TABLE V: Same as Table IV for $b b \bar{n} \bar{n}$ states.

\begin{tabular}{|c|c|c|c|c|c|c|}
\hline & \multicolumn{3}{|c|}{ UN } & \multicolumn{3}{|c|}{$\mathrm{CO}$} \\
\hline$J^{P}(I)$ & $\left.I_{1} M_{2}\right|_{L}$ & $E\left(M_{1}, M_{2}\right.$ & RMS & $\left.M_{1} M_{2}\right|_{L}$ & $E\left(M_{1}, M_{2}\right)$ & \\
\hline \multicolumn{7}{|c|}{$L=0$} \\
\hline $0^{+}(0)$ & $\left.B_{1} B\right|_{P}$ & 11101 & 0.373 & $\left.B_{1} B\right|_{P}$ & 11101 & 0.37 \\
\hline $0^{+}(1)$ & $\left.B B\right|_{S}$ & 10588 & 0.284 & $\left.B B\right|_{S}$ & 10588 & 0.28 \\
\hline $1^{+}(0,1)$ & $\left.B B^{*}\right|_{S}$ & 10612 & 0.287 & $\left.B B^{*}\right|_{S, D}$ & 10612 & 0.28 \\
\hline $2^{+}(0)$ & $\left.B^{*} B_{J}^{*}\right|_{P}$ & 11128 & 0.377 & $\left.B B^{*}\right|_{D}$ & 10612 & $0.28^{\prime}$ \\
\hline $2^{+}(1)$ & $\left.B^{*} B^{*}\right|_{S}$ & 10636 & 0.291 & $\left.B B\right|_{D}$ & 10588 & 0.28 \\
\hline $0^{-}(0)$ & $\left.B_{1} B_{1}\right|_{P}$ & 11614 & 0.462 & $\left.B B^{*}\right|_{P}$ & 10612 & 0.28 \\
\hline $0^{-}(1)$ & $\left.B_{J}^{*} B_{J}^{*}\right|_{P}$ & 11620 & 0.464 & $\left.B B^{*}\right|_{P}$ & 10612 & 0.28 \\
\hline $1^{-}(0)$ & $\left.B_{1} B_{J}^{*}\right|_{P}$ & 11617 & 0.463 & $\left.B B\right|_{P}$ & 10 & 0.28 \\
\hline $1^{-}(1)$ & $\left.B_{1} B_{J}^{*}\right|_{P}$ & 617 & 0.463 & $\left.B B^{*}\right|_{P}$ & 10 & 0.28 \\
\hline $2^{-}(0,1)$ & $\left.B_{J}^{*} B_{J}^{*}\right|_{P}$ & 11620 & 0.464 & $\left.B B^{*}\right|_{P}$ & 10 & 0.28 \\
\hline \multicolumn{7}{|c|}{$L=1 S=0$} \\
\hline & $\left.3\right|_{P}$ & 588 & 0.284 & $\left.B B\right|_{P}$ & & 0.28 \\
\hline $1^{-}(1)$ & $\left.B B_{1}\right|_{S, D}$ & 11101 & 0.373 & $\left.B B^{*}\right|_{P}$ & 10612 & 0.28 \\
\hline \multicolumn{7}{|c|}{$L=1 S=1$} \\
\hline $0^{-}(0,1)$ & $\left.B B^{*}\right|_{P}$ & 10612 & 0.287 & $\left.B B^{*}\right|_{P}$ & 10 & 0.28 \\
\hline $1^{-}(0)$ & $\left.B B^{*}\right|_{P}$ & 10612 & 0.287 & $\left.B B\right|_{P}$ & 10588 & 0.28 \\
\hline $1^{-}(1)$ & $\left.B B^{*}\right|_{P}$ & 10612 & 0.287 & $\left.B B^{*}\right|_{P}$ & 10612 & 0.28 \\
\hline $2^{-}(0,1)$ & $\left.B B^{*}\right|_{P}$ & 10612 & 0.287 & $\left.B B^{*}\right|_{P}$ & 10612 & $0.28^{\prime}$ \\
\hline \multicolumn{7}{|c|}{$L=1 S=2$} \\
\hline $1^{-}(0)$ & $\left.B^{*} B^{*}\right|_{P}$ & 10636 & 0.291 & $\left.B B\right|_{P}$ & 10588 & 0.28 \\
\hline $1^{-}(1)$ & $\left|B^{*} B_{J}^{*}\right|_{S, D}$ & 11128 & 0.377 & $\left.B B^{*}\right|_{P}$ & 10612 & 0.28 \\
\hline $2^{-}(0)$ & $\left.B^{*} B^{*}\right|_{P}$ & 10636 & 0.291 & $\left.B B^{*}\right|_{P}$ & 10612 & $0.28^{\prime}$ \\
\hline $2^{-}(1)$ & $\left|B^{*} B_{J}^{*}\right|_{S, D}$ & 11128 & 0.377 & $\left.B B^{*}\right|_{P}$ & 10612 & 0.28 \\
\hline $3^{-}(0)$ & $\left.B^{*} B^{*}\right|_{P}$ & 10636 & 0.291 & $\left|B^{*} B^{*}\right|_{P}$ & 10636 & 0.29 \\
\hline $3^{-}(1)$ & $\left|B^{*} B_{J}^{*}\right|_{S, D}$ & 11128 & 0.377 & $\left.B_{1} B\right|_{D}$ & 11101 & 0.37 \\
\hline
\end{tabular}


TABLE VI: Same as Table IV for the BCN model.

\begin{tabular}{|c|c|c|c|c|c|c|}
\hline & \multicolumn{3}{|c|}{ UN } & \multicolumn{3}{|c|}{$\mathrm{CO}$} \\
\hline$J^{P}(I)$ & $\left.M_{1} M_{2}\right|_{L}$ & $\left(M_{1}, M\right.$ & RMS & $\left.M_{1} M_{2}\right|_{L}$ & $\left(M_{1}, M_{2}\right.$ & 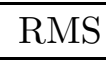 \\
\hline \multicolumn{7}{|c|}{$L=0$} \\
\hline $0^{+}(0)$ & $\left.D_{1} D\right|_{P}$ & 4341 & 0.544 & $\left.D_{1} D\right|_{P}$ & 4341 & 0.54 \\
\hline $0^{+}(1)$ & $\left.D D\right|_{S}$ & 3772 & 0.424 & $\left.D D\right|_{S}$ & 3772 & 0.42 \\
\hline $1^{+}(0,1)$ & $\left.D D^{*}\right|_{S}$ & 3906 & 0.447 & $\left.D D^{*}\right|_{S, D}$ & 3906 & 0.44 \\
\hline $2^{+}(0)$ & $\left.D^{*} D_{J}^{*}\right|_{P}$ & 4511 & 0.577 & $\left.D D^{*}\right|_{D}$ & 3906 & 0.44 \\
\hline $2^{+}(1)$ & $\left.D^{*} D^{*}\right|_{S}$ & 4040 & 0.470 & $\left.D D\right|_{D}$ & 3772 & 0.42 \\
\hline $0^{-}(0)$ & $\left.D_{1} D_{1}\right|_{P}$ & 4910 & 0.664 & $\left.D D^{*}\right|_{P}$ & 3906 & 0.44 \\
\hline $0^{-}(1)$ & $\left.D_{J}^{*} D_{J}^{*}\right|_{P}$ & 4982 & 0.684 & $\left.D D^{*}\right|_{P}$ & 3906 & .44 \\
\hline $1^{-}(0)$ & $\left.D_{1} D_{J}^{*}\right|_{P}$ & 4946 & 0.674 & $\left.D D\right|_{P}$ & 3772 & 42 \\
\hline $1^{-}(1)$ & $\left.D_{1} D_{J}^{*}\right|_{P}$ & 4946 & 0.674 & $\left.D D^{*}\right|_{P}$ & 3906 & 0.44 \\
\hline $2^{-}(0,1)$ & $\left.D_{J}^{*} D_{J}^{*}\right|_{P}$ & 4982 & 0.684 & $\left.D D^{*}\right|_{P}$ & 3906 & 0.44 \\
\hline \multicolumn{7}{|c|}{$L=1 S=0$} \\
\hline $1^{-}(0)$ & $\left.D D\right|_{P}$ & 3772 & 0.424 & $\left.D D\right|_{P}$ & 3772 & 0.42 \\
\hline $1^{-}(1)$ & $\left.D D_{1}\right|_{S, D}$ & 4341 & 0.544 & $\left.D D^{*}\right|_{P}$ & 3906 & 0.44 \\
\hline \multicolumn{7}{|c|}{$L=1 S=1$} \\
\hline $0^{-}(0,1)$ & $\left.D D^{*}\right|_{P}$ & 3906 & 0.447 & $\left.D D^{*}\right|_{P}$ & 3906 & 0.44 \\
\hline $1^{-}(0)$ & $\left.D D^{*}\right|_{P}$ & 3906 & 0.447 & $\left.D D\right|_{P}$ & 3772 & 0.42 \\
\hline $1^{-}(1)$ & $\left.D D^{*}\right|_{P}$ & 3906 & 0.447 & $\left.D D^{*}\right|_{P}$ & 3906 & 0.44 \\
\hline $2^{-}(0,1)$ & $\left.D D^{*}\right|_{P}$ & 3906 & 0.447 & $\left.D D^{*}\right|_{P}$ & 3906 & 0.447 \\
\hline \multicolumn{7}{|c|}{$L=1 S=2$} \\
\hline $1^{-}(0)$ & $\left.D^{*} D^{*}\right|_{P}$ & 4040 & 0.470 & $\left.D D\right|_{P}$ & 3772 & 0.42 \\
\hline $1^{-}(1)$ & $\left.D^{*} D_{J}^{*}\right|_{S, D}$ & 4511 & 0.577 & $\left.D D^{*}\right|_{P}$ & 3906 & 0.44 \\
\hline $2^{-}(0)$ & $\left.D^{*} D^{*}\right|_{P}$ & 4040 & 0.470 & $\left.D D^{*}\right|_{P}$ & 3906 & 0.44 \\
\hline $2^{-}(1)$ & $\left.D^{*} D_{J}^{*}\right|_{S, D}$ & 4511 & 0.577 & $\left.D D^{*}\right|_{P}$ & 3906 & 0.44 \\
\hline $3^{-}(0)$ & $\left.D^{*} D^{*}\right|_{P}$ & 4040 & 0.470 & $\left.D^{*} D^{*}\right|_{P}$ & 4040 & 0.47 \\
\hline $3^{-}(1)$ & $\left.D^{*} D_{J}^{*}\right|_{S, D}$ & 4511 & 0.577 & $\left.D_{1} D\right|_{D}$ & 4341 & 0.54 \\
\hline
\end{tabular}


TABLE VII: Same as Table $\mathrm{V}$ for the BCN model.

\begin{tabular}{|c|c|c|c|c|c|c|}
\hline & \multicolumn{3}{|c|}{$\mathrm{UN}$} & \multicolumn{3}{|c|}{$\mathrm{CO}$} \\
\hline$J^{P}(I)$ & $\overline{\left.I_{1} M_{2}\right|_{L}}$ & $\left(M_{1}, M_{2}\right.$ & $\overline{\mathrm{RMS}}$ & $\left.M_{1} M_{2}\right|_{L}$ & $\left(M_{1}, M_{2}\right)$ & RMS \\
\hline \multicolumn{7}{|c|}{$L=0$} \\
\hline $0+(0)$ & $\left.B_{1} B\right|_{P}$ & 11113 & 0.355 & $\overline{\left.B_{1} B\right|_{P}}$ & 11113 & 0.355 \\
\hline $0^{+}(1)$ & $\left.B B\right|_{S}$ & 10602 & 0.282 & $\left.B B\right|_{S}$ & 10602 & 0.282 \\
\hline $1^{+}(0,1)$ & $\left.B B^{*}\right|_{S}$ & 10651 & 0.288 & $\left.B B^{*}\right|_{S, D}$ & 10651 & 0.288 \\
\hline $2^{+}(0)$ & $\left.B^{*} B_{J}^{*}\right|_{P}$ & 11176 & 0.364 & $\left.B B^{*}\right|_{D}$ & 10651 & 0.288 \\
\hline $2^{+}(1)$ & $\left.B^{*} B^{*}\right|_{S}$ & 10700 & 0.294 & $\left.B B\right|_{D}$ & 10602 & 0.282 \\
\hline $0^{-}(0)$ & $\left.B_{1} B_{1}\right|_{P}$ & 11624 & 0.428 & $\left.B B^{*}\right|_{P}$ & 10651 & 0.288 \\
\hline $0^{-}(1)$ & $\left.B_{J}^{*} B_{J}^{*}\right|_{P}$ & 652 & 0.434 & $\left.B B^{*}\right|_{P}$ & 10 & .288 \\
\hline $1^{-}(0)$ & $\left.B_{1} B_{J}^{*}\right|_{P}$ & 38 & 0.431 & $\left.B B\right|_{P}$ & 10602 & .282 \\
\hline $1^{-}(1)$ & $\left.B_{1} B_{J}^{*}\right|_{P}$ & 11638 & 0.431 & $\left.B B^{*}\right|_{P}$ & 10651 & 0.288 \\
\hline $2^{-}(0,1)$ & $\left.B_{J}^{*} B_{J}^{*}\right|_{P}$ & 652 & 0.434 & $\left.B B^{*}\right|_{P}$ & 10651 & 0.288 \\
\hline \multicolumn{7}{|c|}{$L=1 S=0$} \\
\hline $1^{-}(0)$ & $\left.B B\right|_{P}$ & 10602 & 0.282 & $\left.B B\right|_{P}$ & 10602 & 0.282 \\
\hline $1^{-}(1)$ & $\left.B B_{1}\right|_{S, D}$ & 11113 & 0.355 & $\left.B B^{*}\right|_{P}$ & 10651 & 0.288 \\
\hline \multicolumn{7}{|c|}{$L=1 S=1$} \\
\hline $0^{-}(0,1)$ & $\left.B B^{*}\right|_{P}$ & 10651 & 0.288 & $\left.B B^{*}\right|_{P}$ & 10651 & 0.288 \\
\hline $1^{-}(0)$ & $\left.B B^{*}\right|_{P}$ & 10651 & 0.288 & $\left.B B\right|_{P}$ & 10602 & 0.282 \\
\hline $1^{-}(1)$ & $\left.B B^{*}\right|_{P}$ & 10651 & 0.288 & $\left.B B^{*}\right|_{P}$ & 10651 & 0.288 \\
\hline $2^{-}(0,1)$ & $\left.B B^{*}\right|_{P}$ & 10651 & 0.288 & $\left.B B^{*}\right|_{P}$ & 10651 & 0.288 \\
\hline \multicolumn{7}{|c|}{$L=1 S=2$} \\
\hline $1^{-}(0)$ & $\left.B^{*} B^{*}\right|_{P}$ & 10700 & 0.294 & $\left.B B\right|_{P}$ & 10602 & 0.282 \\
\hline $1^{-}(1)$ & $\left|B^{*} B_{J}^{*}\right|_{S, D}$ & 11176 & 0.364 & $\left.B B^{*}\right|_{P}$ & 10651 & 0.288 \\
\hline $2^{-}(0)$ & $\left.B^{*} B^{*}\right|_{P}$ & 10700 & 0.294 & $\left.B B^{*}\right|_{P}$ & 10651 & 0.288 \\
\hline $2^{-}(1)$ & $\left|B^{*} B_{J}^{*}\right|_{S, D}$ & 11176 & 0.364 & $\left.B B^{*}\right|_{P}$ & 10651 & 0.288 \\
\hline $3^{-}(0)$ & $\left.B^{*} B^{*}\right|_{P}$ & 10700 & 0.294 & $\left.B^{*} B^{*}\right|_{P}$ & 10700 & 0.294 \\
\hline $3^{-}(1)$ & $\left|B^{*} B_{J}^{*}\right|_{S, D}$ & 11176 & 0.364 & $\left.B_{1} B\right|_{D}$ & 11113 & 0.355 \\
\hline
\end{tabular}

TABLE VIII: Comparison among different numerical approaches to the $c c \bar{n} \bar{n}$ system. Energies are in $\mathrm{MeV}$.

\begin{tabular}{|cccc|}
\hline$(L, S, I)$ & Ref. [14] & $\mathrm{HH}\left(\sum_{i} \ell_{i}=0\right)$ & $\mathrm{HH}$ \\
\hline$(0,0,1)$ & 4155 & 4154 & 3911 \\
$(0,1,0)$ & 3927 & 3926 & 3860 \\
$(0,1,1)$ & 4176 & 4175 & 3975 \\
$(0,2,1)$ & 4195 & 4193 & 4031 \\
\hline
\end{tabular}


TABLE IX: BCN and CQC $(L, S, I)=(0,1,0) c c \bar{n} \bar{n}$ results.

\begin{tabular}{|c|cc|cc|}
\hline & \multicolumn{2}{|c|}{ BCN } & \multicolumn{2}{c|}{ CQC } \\
\hline$K$ & $E_{4 q}(\mathrm{MeV})$ & $\mathrm{RMS}(\mathrm{fm})$ & $E_{4 q}(\mathrm{MeV})$ & $\mathrm{RMS}(\mathrm{fm})$ \\
\hline 0 & 4100 & 0.310 & 4109 & 0.314 \\
2 & 3999 & 0.326 & 3990 & 0.320 \\
4 & 3954 & 0.345 & 3931 & 0.331 \\
6 & 3933 & 0.364 & 3903 & 0.341 \\
8 & 3921 & 0.382 & 3887 & 0.348 \\
10 & 3914 & 0.398 & 3878 & 0.354 \\
12 & 3910 & 0.414 & 3872 & 0.358 \\
14 & 3907 & 0.428 & 3868 & 0.361 \\
16 & 3904 & 0.441 & 3866 & 0.363 \\
18 & 3903 & 0.453 & 3864 & 0.365 \\
20 & 3901 & 0.464 & 3862 & 0.366 \\
22 & 3900 & 0.474 & 3861 & 0.367 \\
24 & 3900 & 0.484 & 3861 & - \\
26 & 3899 & 0.492 & - & - \\
\hline$\left.D D^{*}\right|_{S}$ & 3906 & 0.447 & 3937 & 0.454 \\
\hline$\Delta_{E}$ & \multicolumn{3}{|c|}{-7} & \multicolumn{2}{|c|}{0.808} \\
$\Delta_{R}$ & \multicolumn{2}{|c}{} \\
\hline
\end{tabular}


TABLE X: $c c \bar{n} \bar{n}$ CQC energy, $E_{4 q} \equiv E_{4 q}\left(K_{\max }\right)$ (in MeV), RMS (in fm), $\Delta_{R}$, and $\Delta_{E}$ (in MeV) as defined in Eqs. (7) and (10). $\left.M_{1} M_{2}\right|_{L}$ indicates the lowest threshold and $E\left(M_{1}, M_{2}\right)$ its energy as obtained from Table IV.

\begin{tabular}{|cc|cc|cccc|}
\hline$K_{\max }$ & $J^{P}(L, S, I)$ & $\left.M_{1} M_{2}\right|_{L}$ & $E\left(M_{1}, M_{2}\right)$ & $E_{4 q}$ & $\mathrm{RMS}_{4 q}$ & $\Delta_{R}$ & $\Delta_{E}$ \\
\hline 28 & $0^{+}(0,0,0)$ & $\left.D_{1} D\right|_{P}$ & 4426 & 4441 & 0.624 & $>1$ & +15 \\
28 & $0^{+}(0,0,1)$ & $\left.D D\right|_{S}$ & 3872 & 3905 & 0.752 & $>1$ & +33 \\
24 & $1^{+}(0,1,0)$ & $\left.D D^{*}\right|_{S}$ & 3937 & 3861 & 0.367 & 0.808 & -76 \\
24 & $1^{+}(0,1,1)$ & $\left.D D^{*}\right|_{S}$ & 3937 & 3972 & 0.779 & $>1$ & +35 \\
30 & $2^{+}(0,2,0)$ & $\left.D^{*} D_{J}^{*}\right|_{P}$ & 4499 & 4526 & 0.987 & $>1$ & +27 \\
30 & $2^{+}(0,2,1)$ & $\left.D^{*} D^{*}\right|_{S}$ & 4002 & 4025 & 0.879 & $>1$ & +22 \\
& & & & & & & \\
25 & $0^{-}(0,0,0)$ & $\left.D_{1} D_{1}\right|_{P}$ & 4980 & 5374 & 0.738 & $>1$ & +394 \\
25 & $0^{-}(0,0,1)$ & $\left.D_{J}^{*} D_{J}^{*}\right|_{P}$ & 4996 & 5012 & 0.982 & $>1$ & +16 \\
25 & $1^{-}(0,1,0)$ & $\left.D_{1} D_{J}^{*}\right|_{P}$ & 4988 & 5021 & 0.944 & $>1$ & +33 \\
25 & $1^{-}(0,1,1)$ & $\left.D_{1} D_{J}^{*}\right|_{P}$ & 4988 & 5018 & 0.982 & $>1$ & +30 \\
25 & $2^{-}(0,2,0)$ & $\left.D_{J}^{*} D_{J}^{*}\right|_{P}$ & 4996 & 5387 & 1.237 & $>1$ & +391 \\
25 & $2^{-}(0,2,1)$ & $\left.D_{J}^{*} D_{J}^{*}\right|_{P}$ & 4996 & 5025 & 0.991 & $>1$ & +29 \\
& & & & & & & \\
21 & $1^{-}(1,0,0)$ & $D D_{P}$ & 3872 & 3938 & 0.726 & $>1$ & +66 \\
23 & $1^{-}(1,0,1)$ & $\left.D D_{1}\right|_{S, D}$ & 4426 & 4426 & 0.527 & $0.894^{a}$ & +0 \\
21 & $(0,1,2)^{-}(1,1,0)$ & $\left.D D^{*}\right|_{P}$ & 3937 & 3996 & 0.739 & $>1$ & +59 \\
21 & $(0,1,2)^{-}(1,1,1)$ & $\left.D D^{*}\right|_{P}$ & 3937 & 4004 & 0.814 & $>1$ & +67 \\
21 & $(1,2,3)^{-}(1,2,0)$ & $\left.D^{*} D^{*}\right|_{P}$ & 4002 & 4052 & 0.817 & $>1$ & +50 \\
19 & $(1,2,3)^{-}(1,2,1)$ & $\left.D^{*} D_{J}^{*}\right|_{S, D}$ & 4499 & 4461 & 0.465 & 0.766 & -38 \\
\hline
\end{tabular}

${ }^{a}$ In this case the radius has still yet to converge for $K=K_{\max }$. Its extrapolation gives a value larger than one but finite. 
TABLE XI: $c c \bar{n} \bar{n}$ CQC energies and $\Delta_{E}$, in $\mathrm{MeV}$, evaluated for $K_{\max }$ and using the extrapolation (8) in the limit $K \rightarrow \infty$.

\begin{tabular}{|c|cc|cc|}
\hline$J^{P}(L, S, I)$ & $E_{4 q}\left(K_{\max }\right)$ & $\Delta_{E}$ & $E_{4 q}(K=\infty)$ & $\Delta_{E}$ \\
\hline $0^{+}(0,0,0)$ & 4441 & +15 & 4429 & +3 \\
$0^{+}(0,0,1)$ & 3905 & +33 & 3862 & -10 \\
$1^{+}(0,1,0)$ & 3861 & -76 & 3856 & -81 \\
$1^{+}(0,1,1)$ & 3972 & +35 & 3914 & -14 \\
$2^{+}(0,2,0)$ & 4526 & +27 & 4501 & +2 \\
$2^{+}(0,2,1)$ & 4024 & +22 & 3991 & -9 \\
& & & & \\
$0^{-}(0,0,0)$ & 5374 & +394 & 5323 & +343 \\
$0^{-}(0,0,1)$ & 5012 & +16 & 4983 & -13 \\
$1^{-}(0,1,0)$ & 5021 & +33 & 4993 & +5 \\
$1^{-}(0,1,1)$ & 5018 & +30 & 4992 & +4 \\
$2^{-}(0,2,0)$ & 5387 & +391 & 5307 & +311 \\
$2^{-}(0,2,1)$ & 5025 & +29 & 5000 & +4 \\
& & & & \\
$1^{-}(1,0,0)$ & 3938 & +66 & 3865 & -7 \\
$1^{-}(1,0,1)$ & 4426 & +0 & 4420 & -6 \\
$(0,1,2)^{-}(1,1,0)$ & 3996 & +59 & 3927 & -10 \\
$(0,1,2)^{-}(1,1,1)$ & 4004 & +67 & 3918 & -19 \\
$(1,2,3)^{-}(1,2,0)$ & 4052 & +50 & 3993 & -9 \\
$(1,2,3)^{-}(1,2,1)$ & 4461 & -38 & 4461 & -38 \\
\hline
\end{tabular}

TABLE XII: Same as Table X for the $b b \bar{n} \bar{n}$ system.

\begin{tabular}{|cc|cc|cccc|}
\hline$K_{\max }$ & $J^{P}(L, S, I)$ & $\left.M_{1} M_{2}\right|_{L}$ & $E\left(M_{1}, M_{2}\right)$ & $E_{4 q}$ & $\mathrm{RMS}_{4 q}$ & $\Delta_{R}$ & $\Delta_{E}$ \\
\hline 30 & $0^{+}(0,0,0)$ & $\left.B B_{1}\right|_{P}$ & 11101 & 10952 & 0.328 & 0.881 & -149 \\
26 & $0^{+}(0,0,1)$ & $\left.B B\right|_{S}$ & 10588 & 10606 & 0.365 & $>1$ & +18 \\
22 & $1^{+}(0,1,0)$ & $\left.B B^{*}\right|_{S}$ & 10612 & 10398 & 0.220 & 0.765 & -214 \\
24 & $1^{+}(0,1,1)$ & $\left.B B^{*}\right|_{S}$ & 10612 & 10623 & 0.310 & $>1$ & +11 \\
28 & $2^{+}(0,2,0)$ & $\left.B_{J}^{*} B^{*}\right|_{P}$ & 11128 & 11144 & 0.627 & $>1$ & +16 \\
30 & $2^{+}(0,2,1)$ & $\left.B^{*} B^{*}\right|_{S}$ & 10635 & 10636 & 0.314 & $>1$ & +1 \\
& & & & & & & \\
23 & $1^{-}(1,0,0)$ & $\left.B B\right|_{P}$ & 10588 & 10577 & 0.335 & $>1$ & -11 \\
19 & $1^{-}(1,0,1)$ & $\left.B B_{1}\right|_{S, D}$ & 11101 & 10980 & 0.276 & 0.740 & -121 \\
21 & $(0,1,2)^{-}(1,1,0)$ & $\left.B B^{*}\right|_{P}$ & 10612 & 10650 & 0.493 & $>1$ & +38 \\
21 & $(0,1,2)^{-}(1,1,1)$ & $\left.B B^{*}\right|_{P}$ & 10612 & 10666 & 0.517 & $>1$ & +54 \\
21 & $(1,2,3)^{-}(1,2,0)$ & $\left.B^{*} B^{*}\right|_{P}$ & 10635 & 10677 & 0.483 & $>1$ & +42 \\
19 & $(1,2,3)^{-}(1,2,1)$ & $\left.B^{*} B_{J}^{*}\right|_{S, D}$ & 11128 & 10988 & 0.276 & 0.732 & -140 \\
\hline
\end{tabular}


TABLE XIII: Same as Table $\mathrm{X}$ for the BCN model. In all cases the value obtained for the radius has still yet to converge for $K=K_{\max }$.

\begin{tabular}{|cc|cc|cccc|}
\hline$K_{\max }$ & $J^{P}(L, S, I)$ & $\left.M_{1} M_{2}\right|_{L}$ & $E\left(M_{1}, M_{2}\right)$ & $E_{4 q}$ & $\mathrm{RMS}_{4 q}$ & $\Delta_{R}$ & $\Delta_{E}$ \\
\hline 26 & $1^{+}(0,1,0)$ & $\left.D D^{*}\right|_{S}$ & 3906 & 3899 & 0.492 & $>1$ & -7 \\
21 & $1^{-}(1,0,1)$ & $\left.D D_{1}\right|_{S, D}$ & 4341 & 4380 & 0.640 & $>1$ & +39 \\
21 & $(1,2,3)^{-}(1,2,1)$ & $\left.D^{*} D_{J}^{*}\right|_{S, D}$ & 4511 & 4502 & 0.492 & 0.853 & -9 \\
\hline
\end{tabular}

TABLE XIV: Same as Table XIII for the $b b \bar{n} \bar{n}$ system.

\begin{tabular}{|cc|cc|cccc|}
\hline$K_{\max }$ & $J^{P}(L, S, I)$ & $\left.M_{1} M_{2}\right|_{L}$ & $E\left(M_{1}, M_{2}\right)$ & $E_{4 q}$ & $\mathrm{RMS}_{4 q}$ & $\Delta_{R}$ & $\Delta_{E}$ \\
\hline 28 & $0^{+}(0,0,0)$ & $\left.B B_{1}\right|_{P}$ & 11113 & 11061 & 0.334 & 0.941 & -52 \\
28 & $1^{+}(0,1,0)$ & $\left.B B^{*}\right|_{S}$ & 10651 & 10507 & 0.220 & 0.764 & -144 \\
28 & $2^{+}(0,2,1)$ & $\left.B^{*} B^{*}\right|_{S}$ & 10700 & 10723 & 0.353 & $>1$ & +23 \\
19 & $1^{-}(1,0,0)$ & $\left.B B\right|_{P}$ & 10602 & 10639 & 0.433 & $>1$ & +27 \\
19 & $1^{-}(1,0,1)$ & $\left.B B_{1}\right|_{S, D}$ & 11113 & 11037 & 0.265 & 0.746 & -76 \\
19 & $(1,2,3)^{-}(1,2,1)$ & $\left.B^{*} B_{J}^{*}\right|_{S, D}$ & 11176 & 11057 & 0.264 & 0.727 & -119 \\
\hline
\end{tabular}

TABLE XV: Summary of bound states.

\begin{tabular}{|cccc|}
\hline Quark content & $J^{P}(L, S, I)$ & Model & Decay mode \\
\hline$c c \bar{n} \bar{n}$ & $1^{+}(0,1,0)$ & CQC & Weak \\
& & BCN & Electromagnetic \\
\hline$b b \bar{n} \bar{n}$ & $1^{+}(0,1,0)$ & CQC & Weak \\
& & BCN & Weak \\
& $3^{-}(1,2,1)$ & CQC & Electromagnetic \\
& & BCN & Electromagnetic \\
& $0^{+}(0,0,0)$ & CQC & Electromagnetic \\
& & BCN & Electromagnetic \\
& $1^{-}(1,0,0)$ & CQC & Weak \\
\hline
\end{tabular}

TABLE XVI: $\Delta_{E}$, in MeV, in the uncoupled $\left(\Delta_{E}^{\mathrm{UN}}\right)$ and coupled schemes $\left(\Delta_{E}^{\mathrm{CO}}\right)$ for different states with the $\mathrm{CQC}$ and BCN models. See text for details

\begin{tabular}{|cc|cc|cc|cc|cc|}
\hline & \multicolumn{5}{|c|}{ CQC } & \multicolumn{4}{c|}{$\mathrm{BCN}$} \\
\hline & $J^{P}(L, S, I)$ & $\left.M_{1} M_{2}\right|^{L S}$ & $\Delta_{E}^{\mathrm{UN}}$ & $\left.M_{1} M_{2}\right|^{J}$ & $\Delta_{E}^{\mathrm{CO}}$ & $\left.M_{1} M_{2}\right|^{J}$ & $\Delta_{E}^{\mathrm{UN}}$ & $\left.M_{1} M_{2}\right|^{J}$ & $\Delta_{E}^{\mathrm{CO}}$ \\
\hline$c c \bar{n} \bar{n}$ & $1^{-}(1,2,1)$ & 4499 & -38 & 3937 & +524 & 4511 & -9 & 3906 & +596 \\
& $2^{-}(1,2,1)$ & 4499 & -38 & 3937 & +524 & 4511 & -9 & 3906 & +596 \\
& $3^{-}(1,2,1)$ & 4499 & -38 & 4426 & +35 & 4511 & -9 & 4341 & +161 \\
& $1^{-}(1,0,1)$ & 4426 & 0 & 3937 & +489 & 4341 & +39 & 3906 & +474 \\
\hline$b b \bar{n} \bar{n}$ & $1^{-}(1,2,1)$ & 11128 & -140 & 10612 & +376 & 11176 & -119 & 10651 & +406 \\
& $2^{-}(1,2,1)$ & 11128 & -140 & 10612 & +376 & 11176 & -119 & 10651 & +406 \\
& $3^{-}(1,2,1)$ & 11128 & -140 & 11101 & -113 & 11176 & -119 & 11113 & -56 \\
& $1^{-}(1,0,1)$ & 11101 & -121 & 10612 & +368 & 11113 & -76 & 10651 & +386 \\
\hline
\end{tabular}


TABLE XVII: Probability of the lowest threshold $P_{M_{1} M_{2}}$ for different bound and unbound fourquark states. Energies in $\mathrm{MeV}$ and radii in $\mathrm{fm}$.

\begin{tabular}{|ccccccc|}
\hline Quark content & $J^{P}(L, S, I)$ & $E_{4 q}$ & $\Delta_{E}$ & $\mathrm{RMS}$ & $\Delta_{R}$ & $P_{M_{1} M_{2}}$ \\
\hline$c c \bar{n} \bar{n}$ & $0^{+}(0,0,1)$ & 3877 & +5 & 30.49 & 60.29 & 1.00 \\
& $1^{+}(0,1,0)$ & 3861 & -76 & 0.37 & 0.81 & 0.50 \\
\hline$b b \bar{n} \bar{n}$ & $0^{+}(0,0,0)$ & 10948 & -153 & 0.33 & 0.89 & 0.25 \\
& $1^{+}(0,1,0)$ & 10397 & -217 & 0.22 & 0.77 & 0.50 \\
\hline
\end{tabular}

TABLE XVIII: Energies and RMS of the $b b \bar{n} \bar{n} 2^{+}(0,2,1)$ as a function of $K$ for the CQC model with $(A)$ and without $(B)$ boson exchange potentials.

\begin{tabular}{|c|cc|cc|}
\hline & \multicolumn{2}{|c|}{$A$} & \multicolumn{2}{c|}{$B$} \\
\hline$K$ & $E_{4 q}(\mathrm{MeV})$ & $\mathrm{RMS}(\mathrm{fm})$ & $E_{4 q}(\mathrm{MeV})$ & $\mathrm{RMS}(\mathrm{fm})$ \\
\hline 0 & 10763.0 & 0.2131 & 10793.3 & 0.2173 \\
2 & 10701.8 & 0.2201 & 10740.6 & 0.2250 \\
4 & 10670.6 & 0.2283 & 10712.2 & 0.2337 \\
6 & 10658.0 & 0.2360 & 10700.3 & 0.2425 \\
8 & 10650.1 & 0.2436 & 10692.2 & 0.2521 \\
10 & 10645.6 & 0.2509 & 10687.2 & 0.2628 \\
12 & 10642.6 & 0.2580 & 10683.5 & 0.2743 \\
14 & 10640.7 & 0.2647 & 10680.7 & 0.2874 \\
16 & 10639.4 & 0.2712 & 10678.6 & 0.3025 \\
18 & 10638.5 & 0.2775 & 10676.6 & 0.3203 \\
20 & 10637.8 & 0.2836 & 10674.9 & 0.3415 \\
22 & 10637.3 & 0.2897 & 10673.3 & 0.3670 \\
24 & 10636.8 & 0.2957 & 10671.7 & 0.3973 \\
26 & 10636.5 & 0.3021 & 10670.1 & 0.4325 \\
28 & 10636.2 & 0.3077 & 10668.4 & 0.4720 \\
30 & 10636.0 & 0.3136 & 10666.8 & 0.5146 \\
\hline$\left.B^{*} B^{*}\right|_{S}$ & 10635.5 & 0.2906 & 10635.5 & 0.2906 \\
\hline
\end{tabular}


FIG. 3: Possible electromagnetic (upper part) and weak (lower part) decays for $c c \bar{n} \bar{n}$ bound states. The electromagnetic decay goes through an intermediate virtual meson (gray box) whose quantum numbers will depend on those of the initial four-quark state, virtual $D^{*}$ for the case of $J^{P}=1^{+}$. The weak decay showed corresponds to the Cabibbo allowed one into two kaons that incorporates two vertex $(c+\bar{u}) \rightarrow(s+\bar{d})$ or $(c+\bar{d}) \rightarrow(\bar{s}+u)$. Other diagrams involving two $W$ emission into fermion-antifermion, $(c c \bar{q} \bar{q}) \rightarrow(s \bar{q})+(s \bar{q})+(f \bar{f})+(f \bar{f})$, or mesons, $(c c \bar{q} \bar{q}) \rightarrow(s \bar{q})+(s \bar{q})+(u \bar{d})+(u \bar{d})$, may also contribute [41].
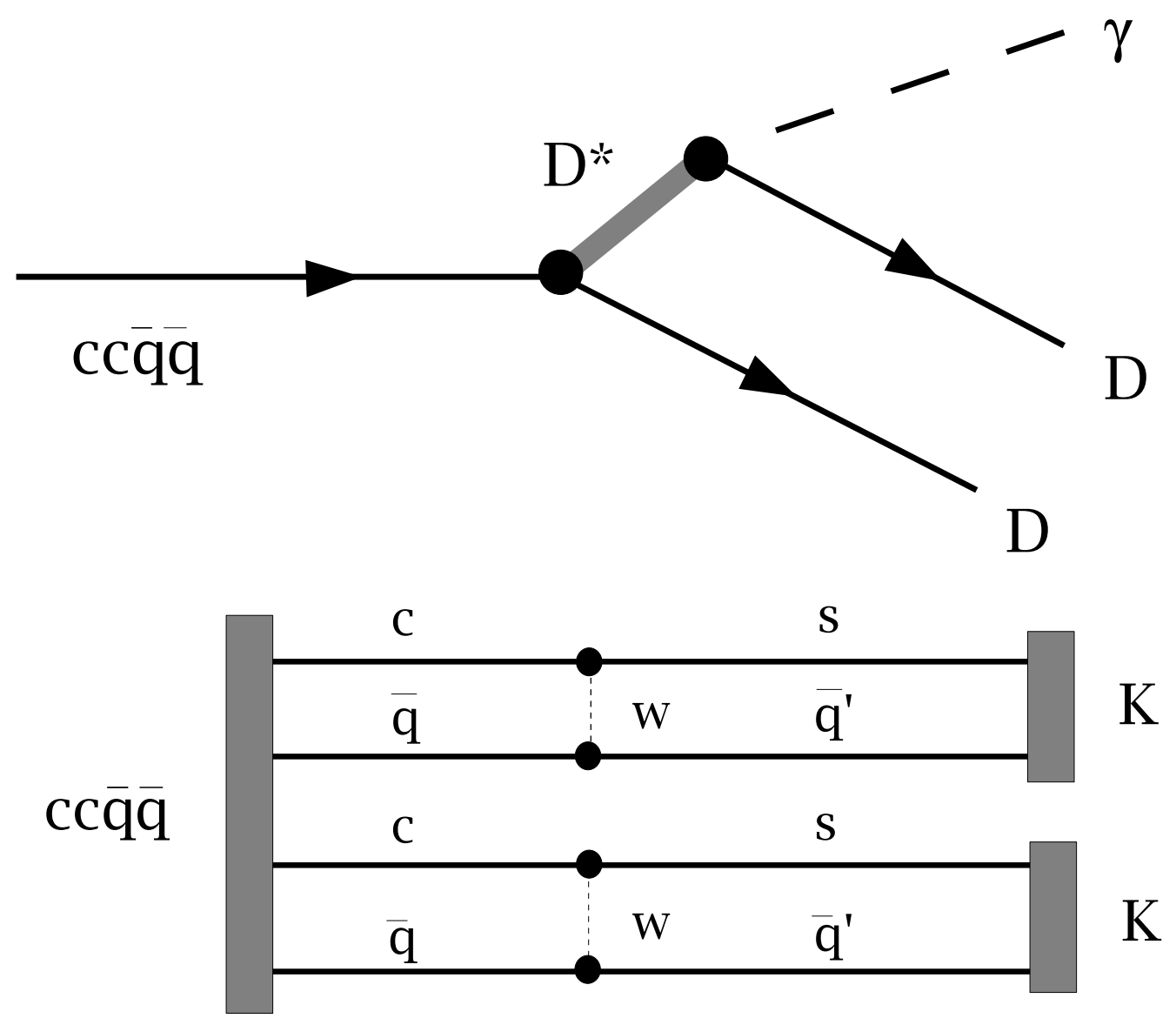
FIG. 4: $\Delta_{E}$ as a function of the heavy quark mass, $m_{Q}$, for $1^{-}(1,0,1)$ with the CQC model. The grey bands take into account the differences between the values obtained for $K_{\max }$, band upper part, and the result obtained with the extrapolation, black squares.

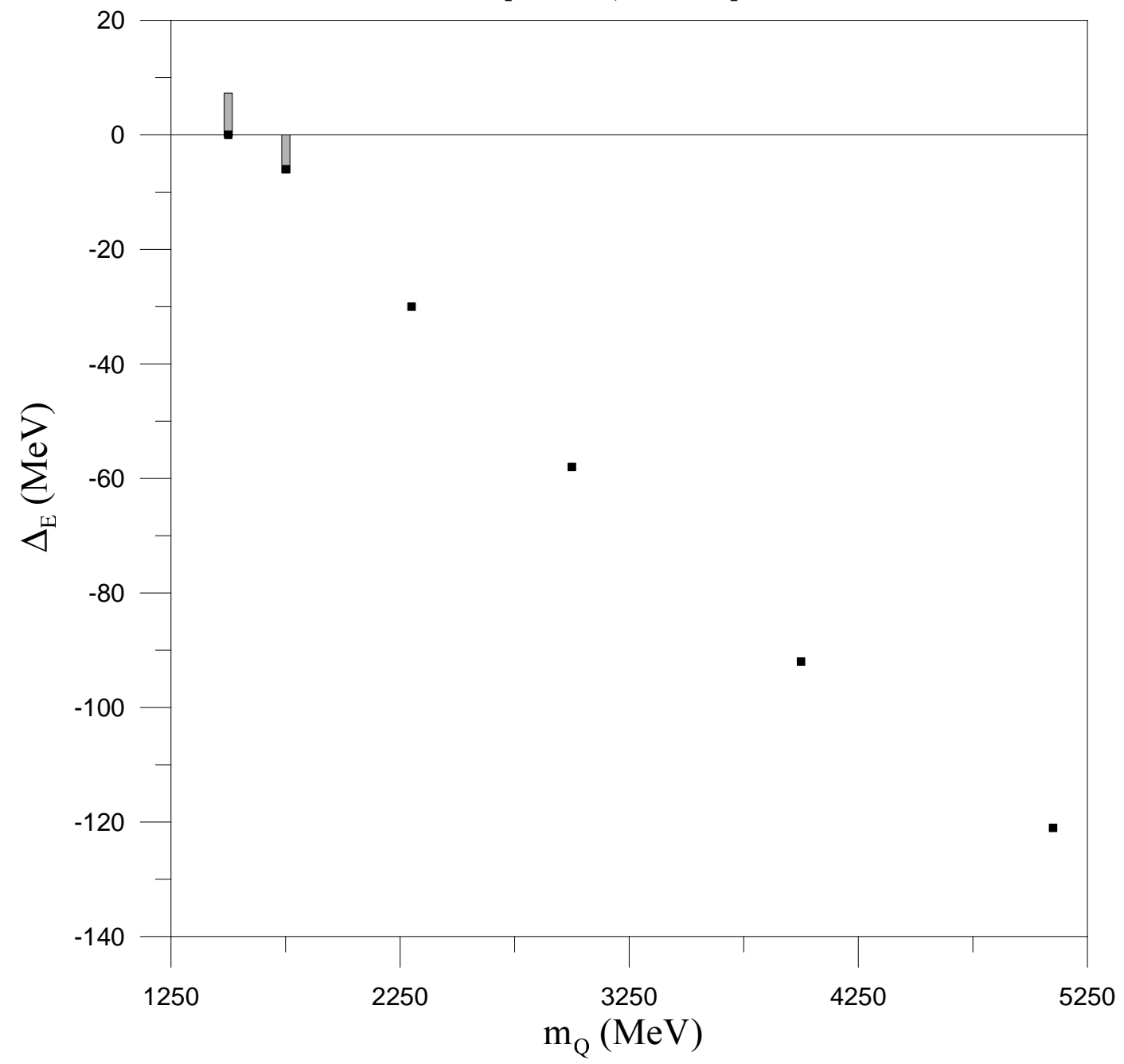


FIG. 5: $\Delta_{R}$ as a function of $K$ for the $2^{+}(0,2,1) b b \bar{n} \bar{n}$ with boson exchange potentials (solid line) and without (dashed line) for the CQC model.

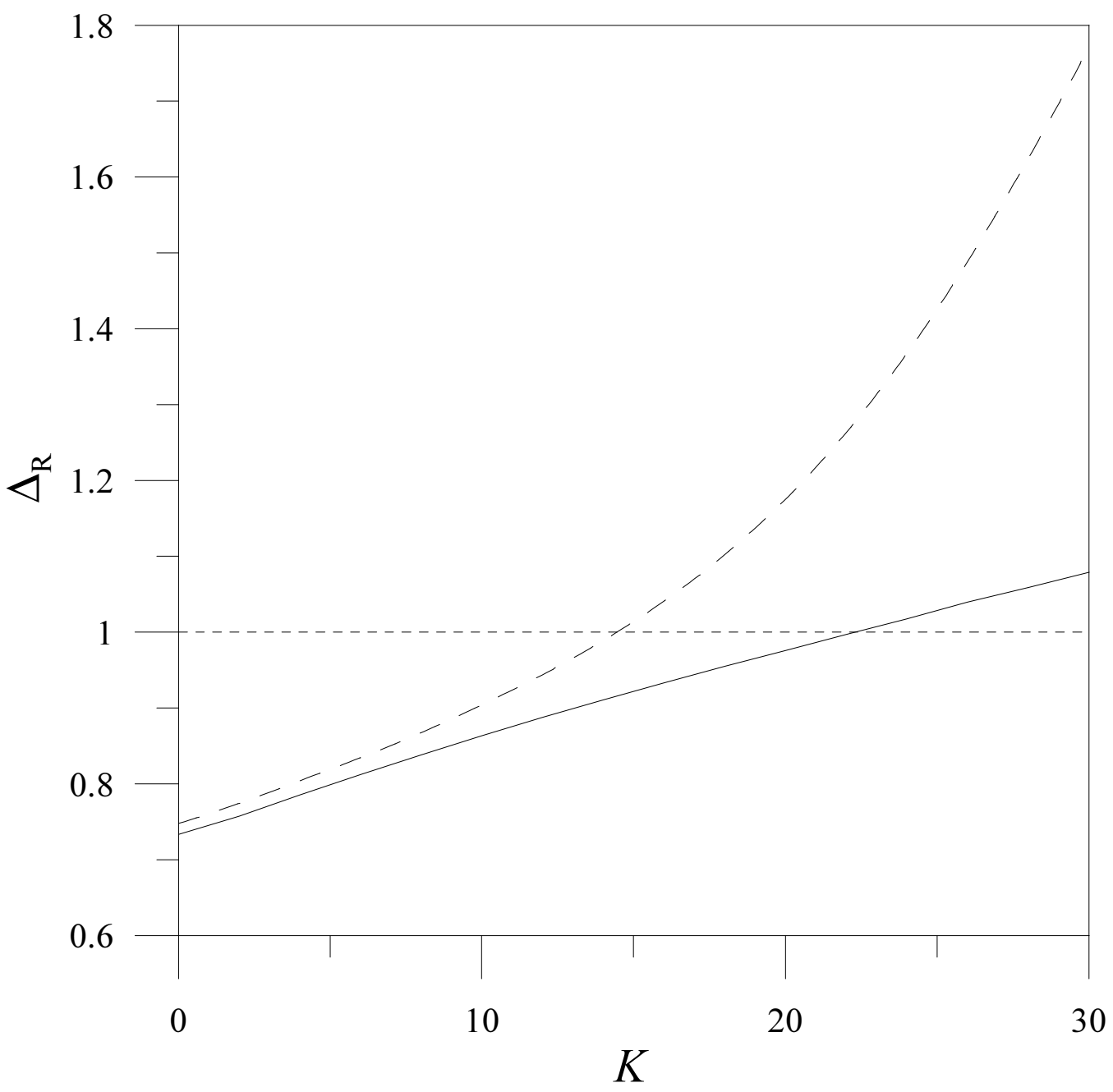

